\title{
An empirically derived three-dimensional Laplace resonance in the Gliese 876 planetary system
}

\author{
Benjamin E. Nelson, ${ }^{1,2 \star}$ Paul M. Robertson, ${ }^{1,2}$ Matthew J. Payne, ${ }^{3}$ Seth M. Pritchard,${ }^{4}$ \\ Katherine M. Deck, ${ }^{5}$ Eric B. Ford,${ }^{1,2}$ Jason T. Wright ${ }^{1,2}$ and Howard T. Isaacson ${ }^{6}$ \\ ${ }^{1}$ Center for Exoplanets and Habitable Worlds, The Pennsylvania State University, 525 Davey Laboratory, University Park, PA 16802, USA \\ ${ }^{2}$ Department of Astronomy \& Astrophysics, The Pennsylvania State University, 525 Davey Laboratory, University Park, PA 16802, USA \\ ${ }^{3}$ Harvard-Smithsonian Center for Astrophysics, 60 Garden Street, Cambridge, MA 02138, USA \\ ${ }^{4}$ Department of Physics \& Astronomy, University of Texas San Antonio, UTSA Circle, San Antonio, TX 78249, USA \\ ${ }^{5}$ Division of Geological and Planetary Sciences, California Institute of Technology, Pasadena, CA 91101, USA \\ ${ }^{6}$ Department of Astronomy, University of California, Berkeley, CA 94720, USA
}

Accepted 2015 October 8. Received 2015 October 5; in original form 2015 April 24

\begin{abstract}
We report constraints on the three-dimensional orbital architecture for all four planets known to orbit the nearby $\mathrm{M}$ dwarf Gliese 876 based solely on Doppler measurements and demanding long-term orbital stability. Our data set incorporates publicly available radial velocities taken with the ELODIE and CORALIE spectrographs, High Accuracy Radial velocity Planet Searcher (HARPS), and Keck HIgh Resolution Echelle Spectrometer (HIRES) as well as previously unpublished HIRES velocities. We first quantitatively assess the validity of the planets thought to orbit GJ 876 by computing the Bayes factors for a variety of different coplanar models using an importance sampling algorithm. We find that a four-planet model is preferred over a three-planet model. Next, we apply a Newtonian Markov chain Monte Carlo algorithm to perform a Bayesian analysis of the planet masses and orbits using an $N$-body model in three-dimensional space. Based on the radial velocities alone, we find that a 99 per cent credible interval provides upper limits on the mutual inclinations for the three resonant planets ( $\Phi_{\mathrm{cb}}<6.20$ for the $c$ and $b$ pair and $\Phi_{\mathrm{be}}<28.5$ for the $b$ and $e$ pair). Subsequent dynamical integrations of our posterior sample find that the GJ 876 planets must be roughly coplanar $\left(\Phi_{\mathrm{cb}}<2.60\right.$ and $\left.\Phi_{\mathrm{be}}<7.87\right)$, suggesting that the amount of planet-planet scattering in the system has been low. We investigate the distribution of the respective resonant arguments of each planet pair and find that at least one argument for each planet pair and the Laplace argument librate. The libration amplitudes in our three-dimensional orbital model support the idea of the outer three planets having undergone significant past disc migration.
\end{abstract}

Key words: methods: numerical - methods: statistical - techniques: radial velocities - planets and satellites: dynamical evolution and stability - planets and satellites: formation.

\section{INTRODUCTION}

Gliese 876 (=GJ 876) is a $0.37 \mathrm{M}_{\odot} \mathrm{M} 4 \mathrm{~V}$ star (von Braun et al. 2014) hosting four known planets. This remarkable system represents several milestones: the first detection of a planet around an M dwarf (GJ 876 b; Delfosse et al. 1998; Marcy et al. 1998), the first example of a multi-planet system orbiting in a mean-motion resonance (MMR; Marcy et al. 2001), the first example of a MMR chain amongst three planets (Rivera et al. 2010), and the closest multi-planet exosystem to date (4.689 pc; van Leeuwen 2007).
The star has a lengthy Doppler (or radial velocity, RV) history spanning two decades and multiple observing sites. Planet $b$ was detected contemporaneously by Marcy et al. (1998) using the Lick Hamilton Spectrograph and Keck HIRES and Delfosse et al. (1998) using the ELODIE and CORALIE spectrographs. Both estimated a moderate eccentricity for $b(\sim 0.3)$ and an orbital period of $61 \mathrm{~d}$ for this gas giant from their RV model. With more RV observations, Marcy et al. (2001) uncovered a second gas giant, $c$, orbiting near $30 \mathrm{~d}$. This planet's RV signature previously masqueraded as a larger eccentricity for planet $b$ due to the near $2: 1$ period commensurability of their orbits (Anglada-Escudé, López-Morales \& Chambers 2010; Ford, Moorhead \& Veras 2011). As the Keck RV data set grew, Rivera et al. (2005) revealed a third planet $d$ orbiting near $1.9 \mathrm{~d}$ and 
was the lowest mass exoplanet around a main-sequence star at the time $\left(m \sin i=5.89 \mathrm{M}_{\oplus}\right)$. Photometric measurements showed planet $d$ did not transit (Laughlin et al. 2005; Rivera et al. 2005; Shankland et al. 2006; Kammer et al. 2014). Correia et al. (2010) published new High Accuracy Radial velocity Planet Searcher (HARPS) RVs which by themselves could constrain the mutual inclination between planets $c$ and $b$. Around the same time, Rivera et al. (2010) published new Keck RVs which showed an additional RV signal around $124 \mathrm{~d}$, dubbed planet $e$. Numerically integrating their solutions beyond the last observation, the outer three planets $(c, b$, and $e$ ) appear to be in a Laplace resonance, much like the three closest Galilean moons orbiting Jupiter. Other studies have placed limits on the existence of additional planets and massive companions in the system through observations (Leinert et al. 1997; Luhman \& Jayawardhana 2002; Patience et al. 2002) and considerations of long-term dynamical stability (Jones, Sleep \& Chambers 2001; Ji \& Liu 2006; Rivera \& Haghighipour 2007; Gerlach \& Haghighipour 2012).

For RV systems, we only observe the component of the planetaryinduced stellar wobble projected on to our line of sight. Most of the time, there is a degeneracy between the true mass $(m)$ and on-sky inclination $(i)$, where an edge-on system is $i_{\text {sys }}=90^{\circ}$, so we can only place a lower limit on the orbiting companion's mass. However, if the self-interactions in a multi-planet system are strong, the RV model becomes sensitive to the true masses of the planets, thereby breaking the $m \sin i_{\text {sys }}$ degeneracy. There are many RV systems where the true masses can be meaningfully constrained, including HD 200964, 24 Sextantis (Johnson et al. 2011), HD 82943 (Tan et al. 2013), and other dynamically active systems (Veras \& Ford 2010).

For GJ $876 c$ and $b$, Laughlin \& Chambers (2001) and Rivera \& Lissauer (2001) performed self-consistent Newtonian fits and constrained the planetary masses and their on-sky coplanar inclination. Rivera et al. (2005) found an on-sky inclination for the three-planet system $\left(i_{\text {sys }}=50^{\circ} \pm 3^{\circ}\right)$, assuming coplanarity. Bean \& Seifahrt (2009) combined the RVs from Rivera et al. (2005) and Hubble Space Telescope (HST) astrometry from Benedict et al. (2002) and measured the mutual inclination $\Phi$ between $c$ and $b$, where

$\cos \Phi_{\mathrm{cb}}=\cos i_{c} \cos i_{b}+\sin i_{c} \sin i_{b} \cos \left(\Omega_{c}-\Omega_{b}\right)$,

$\Omega$ is the longitude of ascending node, and $c$ and $b$ denote the two planets considered. They find $\Phi_{\mathrm{cb}}=5.0 \pm_{2.3}^{3.9}$. Based on the HARPS RVs alone, Correia et al. (2010) find a lower value $\left(\Phi_{\mathrm{cb}}=1.00\right)$. With their four-planet model, Rivera et al. (2010) find a best-fitting value $\Phi_{\mathrm{cb}}=3.7$. All of the above values are generally consistent with a nearly coplanar system. When incorporating the effects of correlated noise, Baluev (2011) places an upper limit of $\Phi_{\mathrm{cb}}=$ $5^{\circ}-15^{\circ}$.

Arguably the most studied exoplanet system displaying an MMR, the GJ 876 system has been a prime test bed for dynamical and planet formation theory for the past decade. The 2:1 MMR of the $c$ and $b$ pair is of particular interest, since the libration amplitudes of the resonant arguments are a valuable indicator of the system's long-term dynamical stability (Kinoshita \& Nakai 2001; Goździewski, Bois \& Maciejewski 2002; Ji, Li \& Liu 2002; Beaugé, Ferraz-Mello \& Michtchenko 2003; Haghighipour et al. 2003; Zhou $\&$ Sun 2003). The most likely mechanism for the planets' current orbital periods and eccentricities is through a combination of planet-planet interactions and disc migration, while most studies have focused on migration through a gas disc (Snellgrove, Papaloizou \& Nelson 2001; Lee \& Peale 2002; Murray, Paskowitz \& Holman 2002; Kley, Peitz \& Bryden 2004; Lee 2004; Kley et al.
2005; Thommes 2005; Thommes, Matsumura \& Rasio 2008; Lee \& Thommes 2009; Podlewska-Gaca, Papaloizou \& Szuszkiewicz 2012; Batygin \& Morbidelli 2013; Lega, Morbidelli \& Nesvorný 2013). (Semi)-analytic models describing the secular evolution of the system have also been developed (Beaugé et al. 2003; Veras 2007).

Other studies of the GJ 876 system include the search for debris discs, thermal properties of $d$, and interior structure models of $d$ (Rivera et al. 2010, and references therein). More recent analyses include how the star's UV radiation field affects habitability (France et al. 2012, 2013) and the detectability of additional hypothesized planets in the system (Gerlach \& Haghighipour 2012). Although the system's orbital architecture appears atypical, GJ 876 does fit into a larger portrait of exoplanet systems around $\mathrm{M}$ dwarfs, including population statistics of giant planets (Montet et al. 2014) and the coplanarity of multi-planet systems (Ballard \& Johnson 2014).

RV surveys have discovered a couple dozen strongly interacting multi-planet systems, motivating the need for analysis procedures to incorporate a self-consistent Newtonian model. Nelson, Ford \& Payne (2014a) developed a Newtonian Markov chain Monte Carlo (MCMC) algorithm which has been successful in analysing systems that require a large number of model parameters (e.g. 55 Cancri; Nelson et al. 2014b). The GJ 876 observations have a similar observing baseline and require almost as many model parameters, making it compatible with such an algorithm.

In this work, we present a detailed characterization of the orbits and masses of the GJ 876 planets employing a full three-dimensional (3D) orbital model used to fit the Doppler observations. In Section 2, we describe the RV observations made with multiple spectrographs (ELODIE, CORALIE, HARPS, and HIRES), including a new set of HIRES measurements. In Section 3, we describe our orbital and observational model, and investigate the effects of correlated noise in the observations. In Section 4, we report our results for a coplanar orbital model. Before advancing to a more complex orbital model, we evaluate the evidence for planets $d$ and $e$ by computing Bayes factors as described in Section 3.4. In Section 5, we report the results of the fitting and $\mathrm{N}$-body simulations for a 3D orbital model. In Section 6, we investigate the evolution of the resonant angles and statistics regarding their libration amplitude. In Appendix A, we test for possible observational biases in these results. We conclude with a discussion of the key results and the applications of our posterior samples in Section 7.

\section{OBSERVATIONS}

Our data set includes publicly available RVs from four different instruments. We include 46 ELODIE, 40 CORALIE, and 52 HARPS observations (Correia et al. 2010). The inclusion of these data extends our observing baseline to roughly $580 \mathrm{~d}$ before the first Keck HIRES observation. The 162 Keck observations are reduced by the Carnegie Planet Search group (Rivera et al. 2010). These will be referred to as the Carnegie RVs henceforth.

Our analysis also includes 67 additional Doppler measurements from HIRES reduced by the California Planet Search group (Table 1). These will be referred to as the California RVs henceforth. We measured relative RVs of GJ 876 with the HIRES echelle spectrometer (Vogt et al. 1994) on the $10 \mathrm{~m}$ Keck I telescope using standard procedures. Most observations were made with the B5 decker $(3.5 \times 0.86$ arcsec $)$. Light from the telescope passed through a glass cell of molecular iodine cell heated to $50^{\circ} \mathrm{C}$. The dense set of molecular absorption lines imprinted on the stellar spectra between 5000 and $6200 \AA$ provide a robust wavelength scale against 
Table 1. New Keck HIRES velocities for GJ 876. This table is presented in its entirety as Supporting Information with the online version of the paper. This sub-table is shown for guidance regarding its form and content.

\begin{tabular}{lcc}
\hline BJD-2450000. (d) & RV $\left(\mathrm{m} \mathrm{s}^{-1}\right)$ & Uncertainty $\left(\mathrm{m} \mathrm{s}^{-1}\right)$ \\
\hline 3301.808032 & -33.89 & 2.06 \\
3301.816875 & -36.49 & 2.37 \\
3301.822986 & -28.19 & 2.60 \\
3301.871412 & -39.54 & 1.99 \\
3302.722523 & -91.03 & 1.91 \\
3302.728866 & -89.20 & 2.02 \\
3302.735567 & -81.93 & 1.92 \\
\hline
\end{tabular}

which Doppler shifts are measured, as well as strong constraints on the instrumental profile at the time of each observation (Marcy \& Butler 1992; Valenti, Butler \& Marcy 1995). We also obtained five iodine-free 'template' spectra using the B1 decker $(3.5 \times 0.57$ $\operatorname{arcsec})$. These spectra were de-convolved using the instrumental profile measured from spectra of rapidly rotating B stars observed immediately before and after through the iodine cell. We measured high-precision relative RVs using a forward model where the de-convolved stellar spectrum is Doppler shifted, multiplied by the normalized high-resolution iodine transmission spectrum, convolved with an instrumental profile, and matched to the observed spectra using a Levenberg-Marquardt algorithm that minimizes the $\chi^{2}$ statistic (Butler et al. 1996). In this algorithm, the RV is varied (along with nuisance parameters describing the wavelength scale and instrumental profile) until the $\chi^{2}$ minimum is reached. Each $\mathrm{RV}$ uncertainty is the weighted error on the mean $\mathrm{RV}$ of $\sim 700 \mathrm{spec}-$ tral chunks that are separately Doppler analysed. These uncertainty estimates do not account for potential systematic Doppler shifts from instrumental or stellar effects.

\section{METHODS}

We characterize the masses and orbits of the GJ 876 planets using the GPU version of the Radial velocity Using $N$-body Differential evolution Markov Chain Monte Carlo code, RUN DMC (Nelson et al. 2014a), which incorporates the SWARM-NG framework to integrate planetary systems on graphics cards (Dindar et al. 2013). RUN DMC has analysed the 55 Cancri planetary system, which required a highdimensional ( 40) model. Compared with 55 Cancri, the problem of GJ 876 seems to be similarly challenging but more computationally tractable due to having fewer observations, a shorter observing baseline, a larger integration time step, and a lower dimensional model (e.g. one less planet to account for). On the other hand, the presence of extremely strong planet-planet interactions can result in a challenging posterior distribution that could be more difficult to sample from.

Considering the lessons from Nelson et al. (2014a) regarding how to explore parameter space efficiently, we set the following algorithmic parameters for RUN DMC: $n_{\text {chains }}=300, \sigma_{\gamma}=0.05$, and MassScaleFactor $=1.0$. To accommodate the innermost planet's $1.9 \mathrm{~d}$ orbital period, we set our integration time step to roughly 17 min and use the time-symmetrized Hermite integrator (Kokubo, Yoshinaga \& Makino 1998).

We begin by applying RUN DMC to the Carnegie RVs from Rivera et al. (2010) for a four-planet model. We sampled from the Markov chains after they had burned-in sufficiently. In RUN DMC, we specify the orbital parameters at the epoch of the first observation in our data set. For the full RV data set, this happens to be an ELODIE observation. To generate the initial states of the Markov chains for analysing the full data set, we start from a posterior sample based on the Carnegie RVs and rewind each planet's argument of periastron $(\omega)$ and mean anomaly $(M)$ according to the precession rate $(\dot{\varpi})$, orbital periods, and time difference between the first ELODIE and HIRES observations. Laughlin et al. (2005) find the joint line of apses for the $c$ and $b$ pair to be precessing at an average rate of $\dot{\varpi}=-41^{\circ} \mathrm{yr}^{-1}$. After burning-in, we randomly sample from our Markov chains to use as our set of initial conditions for long-term stability simulations using the MERCURY hybrid integrator (Chambers 1999). The solutions vetted for stability are used as initial conditions for more restricted RUN DMC runs to be explained in Section 5.

\subsection{Model parameters}

We characterize the system model with a fixed star mass $\left(M_{\star}=\right.$ $0.37 \mathrm{M}_{\odot}$; von Braun et al. 2014), plus each planet's mass $(m)$, semimajor axis $(a)$, eccentricity $(e)$, inclination $(i)$, argument of periastron $(\omega)$, longitude of ascending node $(\Omega)$, and mean anomaly $(M)$ at our chosen epoch (first ELODIE observation) for each planet, plus the RV zero-point offsets $(C)$ and jitters (i.e. unmodelled instrumental and astrophysical noise, $\sigma_{\text {jit }}$ ) for each observatory. We report the orbital periods $(P)$ based on Kepler's Third Law and each body's $m$ and $a$ based in a Jacobi coordinate system. Planet masses and semimajor axes can be readily rescaled to account for any updates to the stellar mass.

The GJ 876 planets are well approximated by a coplanar system, i.e. $\Omega=0^{\circ}$ and $i$ is the same for all planets. Due to the symmetrical nature of an RV system on the sky, a planetary system at $i_{\text {sys }}$ is indistinguishable from one at an inclination of $180^{\circ}-i_{\text {sys. }}$. So in Section 4, we restrict the planets to a coplanar system that can take on any value of $0^{\circ}<i_{\text {sys }}<90^{\circ}$. Similarly, the entire system can be rotated about the line of sight. In Section 5, the individual $i$ and $\Omega$ for each planet become free parameters in our model that are fitted to the observations. We set $\Omega_{d}=0^{\circ}$ to ground our coordinate system.

\subsection{Model of observations}

RUN DMC allows for fitting multiple zero-point offsets and magnitudes of jitter (e.g. combined astrophysical and/or instrumental noise). HIRES received a CCD upgrade and new Doppler reduction process in 2004 August (JD 245 3241.5). The Carnegie time series was split based on this pre- and post-upgrade era, which was modelled by two zero-point offsets, but the entire data set is still modelled with one jitter term. The California RVs also have a separate offset and one jitter term. The other instruments (ELODIE, CORALIE, HARPS) were each modelled by one RV zero-point offset and jitter term. In total, we account for six offsets and five jitter parameters.

Our data set does not include the HST astrometry from Benedict et al. (2002) or any informative priors regarding the inclinations of the GJ 876 planets.

\subsection{Magnitude and time-scale for correlated noise}

Our likelihood function described in equations 4 and 5 of Nelson et al. (2014a) assumes that the observational errors are uncorrelated, which may not be a sufficient approximation for high-precision RV measurements of stars with significant stellar activity. Baluev (2011) showed that correlated ( = red) noise in the RV data could lead to bias or misestimated uncertainty in some of the orbital parameters (e.g. $e_{d}$ ). Recently, some discoveries of planets orbiting M dwarfs have been shown to be more likely mere artefacts resulting from 


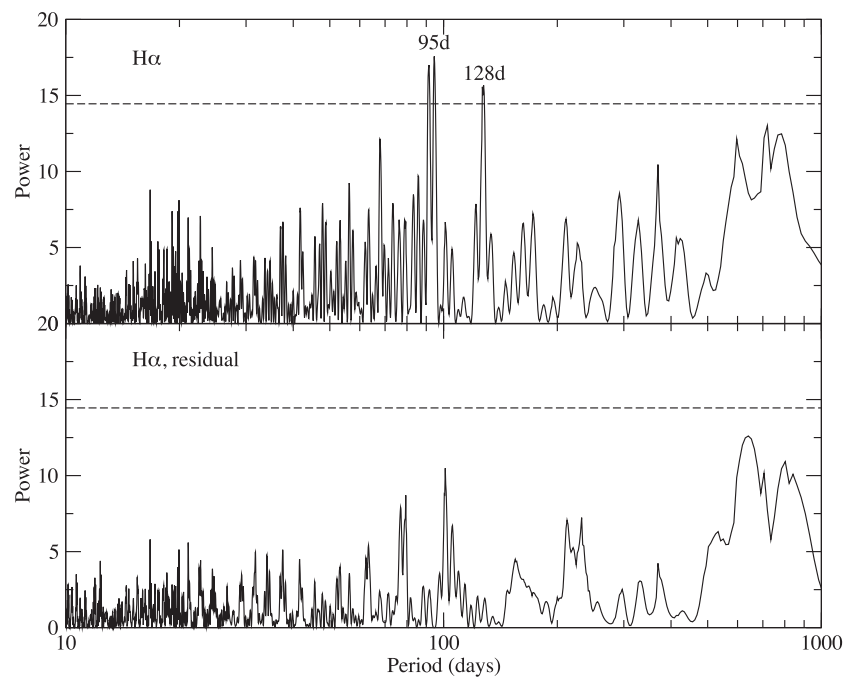

Figure 1. Top: generalized Lomb-Scargle periodogram (Zechmeister \& Kürster 2009) of the $\mathrm{H} \alpha$ stellar activity index for GJ 876, as measured from the publicly available HARPS spectra. We mark the peak at $P=95 \mathrm{~d}$ - which we adopt as the stellar rotation period - and its $1 \mathrm{yr}$ alias at $128 \mathrm{~d}$. Bottom: residual periodogram of $\mathrm{H} \alpha$ after modelling and removing a sinusoid at the rotation period. The dashed lines indicate the power required for a false alarm probability of 1 per cent according to equation 24 of Zechmeister \& Kürster (2009).

stellar activity (Robertson \& Mahadevan 2014; Robertson et al. 2014). Therefore, we take a closer look at the role of stellar activity in contributing astrophysical noise to RV observations of GJ 876. In Section 3.4, we perform a complementary analysis by computing Bayes factors for a finite set of models.

High-precision photometry of GJ 876 (Rivera et al. 2005) revealed a rotation period of $97 \pm 1 \mathrm{~d}$. Examining the variability of the activity-sensitive $\mathrm{H} \alpha$ and $\mathrm{Na}$ ID absorption lines in the publicly available HARPS spectra of the star, ${ }^{1}$ we confirm the rotation period, finding an $\mathrm{H} \alpha$ periodicity of $95 \pm 1 \mathrm{~d}$ (Fig. 1, top panel). The appearance of the rotation period in the photometry suggests the presence of starspots, which can affect the measured RVs (e.g. Boisse et al. 2011; Dumusque, Boisse \& Santos 2014). The results from Baluev (2011), Rivera et al. (2005), and our own examination of the spectral activity tracers suggest that a complete treatment of activity will yield only marginal improvements in the accuracy of the RV model, and is not necessary to achieve the goals of this study.

Boisse et al. (2011) showed that rotating starspots will induce RV periodicities at the stellar rotation period $P_{\text {rot }}$ and its integer fractions $\left(P_{\text {rot }} / 2, P_{\text {rot }} / 3\right.$, etc. $)$. Given that none of the planets in the GJ 876 system have periods near the rotation period or its integer fractions, we conclude that rotating starspots have not produced large-amplitude periodic RV signals such as might be misinterpreted as planet candidates. Although the RV amplitude of the outermost planet $e\left(3.5 \mathrm{~m} \mathrm{~s}^{-1}\right)$ is closest to the amplitudes expected for activity signals produced by a chromospherically quiet star such as GJ 876, we are unaware of any physical mechanism that would create an RV signal on this time-scale (124 days).

Our computed stellar rotation period of $95 \pm 1 \mathrm{~d}$ beating with one Earth year produces an alias of around $128 \mathrm{~d}$, which is worryingly close to the orbital period of planet $e(\sim 124 \mathrm{~d})$. However, subtracting the rotation signal causes the $128 \mathrm{~d}$ peak to disappear as well, leading

\footnotetext{
${ }^{1}$ Based on data obtained from the ESO Science Archive Facility under request number 129169 .
}

us to suspect that this signal is an alias (Fig. 1, bottom panel). If the $124 \mathrm{~d}$ signal is a $1 \mathrm{yr}$ alias of the rotation period, then we would also see the $95 \mathrm{~d}$ signal in the RV data. Rivera et al. (2010) and Baluev (2011) did not find the stellar rotation signal in the RV data sets they analysed.

\subsection{Computing Bayes factors for model selection}

When Doppler observations are taken of a system with multiple strongly interacting planets, the shape of the posterior distribution is often challenging to sample from efficiently. A general Bayesian approach of performing model comparison is to compute the fully marginalized likelihood, sometimes called the evidence, for each model. Formally, the evidence is the probability of generating the observed RV data set $\boldsymbol{d}$ assuming some underlying model $\mathcal{M}$ that is parametrized by $\boldsymbol{\theta}$,

$p(\boldsymbol{d} \mid \mathcal{M})=\int p(\boldsymbol{d} \mid \boldsymbol{\theta}, \mathcal{M}) p(\boldsymbol{\theta} \mid \mathcal{M}) d \boldsymbol{\theta}$,

where $p(\boldsymbol{d} \mid \boldsymbol{\theta}, \mathcal{M})$ is the likelihood function and $p(\boldsymbol{\theta} \mid \mathcal{M})$ is the prior probability distribution. While the value of $p(\boldsymbol{d} \mid \mathcal{M})$ is not useful by itself, the ratio of two evidences for two competing models $\mathcal{M}_{1}$ and $\mathcal{M}_{2}$, yields the Bayes factor

$\mathrm{BF}=\frac{p\left(\boldsymbol{d} \mid \mathcal{M}_{2}\right)}{p\left(\boldsymbol{d} \mid \mathcal{M}_{1}\right)}$

that provides a quantitative measure of which model is preferred and to what degree.

Marginal likelihoods are notoriously difficult to compute. Integrating equation (2) analytically may be possible for some idealized problems with one to a few dimensions, but the model required to describe a $3+$ planet system needs roughly 20 or more parameters. Numerical integration techniques such as Monte Carlo integration become vastly inefficient with increasing dimensionality.

Therefore, we use importance sampling, a more general form of Monte Carlo integration, to calculate the integral in equation (2) and make this problem computationally tractable. Following Ford $\&$ Gregory (2007), we sample from a distribution $g(\boldsymbol{\theta})$ with a known normalization. We multiply the numerator and denominator of the integrand in equation (2) by $g(\boldsymbol{\theta})$,

$p(\boldsymbol{d} \mid \mathcal{M})=\int \frac{p(\boldsymbol{d} \mid \boldsymbol{\theta}, \mathcal{M}) p(\boldsymbol{\theta} \mid \mathcal{M})}{g(\boldsymbol{\theta})} g(\boldsymbol{\theta}) d \boldsymbol{\theta}$.

While the value of $p(\boldsymbol{d} \mid \mathcal{M})$ has not changed, equation (4) can be estimated by drawing $N$ samples from $g(\boldsymbol{\theta})$,

$\widehat{p(\boldsymbol{d} \mid \mathcal{M})}=\frac{1}{N} \sum_{\boldsymbol{\theta}_{i} \sim g(\boldsymbol{\theta})} \frac{p\left(\boldsymbol{d} \mid \boldsymbol{\theta}_{i}, \mathcal{M}\right) p\left(\boldsymbol{\theta}_{i} \mid \mathcal{M}\right)}{g\left(\boldsymbol{\theta}_{i}\right)}$.

The key aspect of having importance sampling work efficiently is to pick an appropriate $g(\boldsymbol{\theta})$. Assuming that our parameter space contains one dominant posterior mode, we choose a multivariate normal with mean vector $\boldsymbol{\mu}$ and covariance matrix $\boldsymbol{\Sigma}$ for $g(\boldsymbol{\theta})$. For each model considered, we will estimate $\boldsymbol{\mu}$ and $\boldsymbol{\Sigma}$ from the coplanar MCMC runs described in Section 4. Our parametrization for $g(\boldsymbol{\theta})$ is $P, K, e \sin \omega, e \cos \omega$, and $\omega+M$ for each planet, the system's orbital inclination $i_{\text {sys }}$, and one $\sigma_{\text {jit }}$ for each observatory. Since we are only interested in computing ratios of $\widehat{p(\boldsymbol{d} \mid \mathcal{M})}$, the priors in zero-point offsets in the calculation will cancel out.

One good strategy with importance sampling is to pick a $g(\boldsymbol{\theta})$ that is heavier in the tails than $p(\boldsymbol{d} \mid \boldsymbol{\theta}, \mathcal{M}) p(\boldsymbol{\theta} \mid \mathcal{M})$. This makes it easier to sample from low-probability parts of the posterior distribution and prevents any samples from resulting in extremely large weights. 


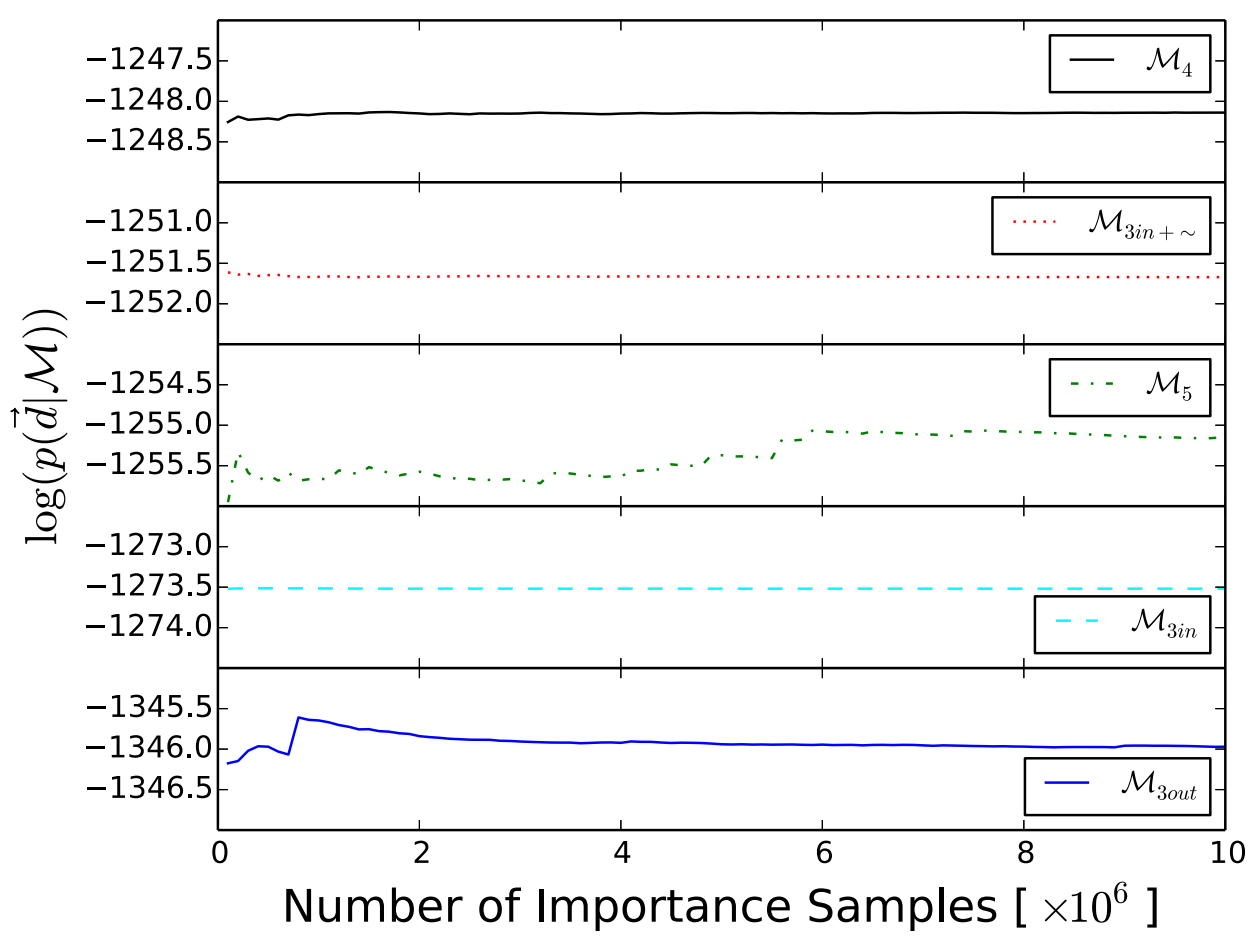

Figure 2. The estimate of $\log (p(\boldsymbol{d} \mid \mathcal{M}))$ (see equation 7) as a function of the number of importance samples for five different models described in Section 4 and Table 2. Each panel shows the convergence of one model and are ranked from least probable (bottom) to most probable (top): $\mathcal{M}_{3 \text { out }}$ (blue solid), $\mathcal{M}_{3 \text { in }}$

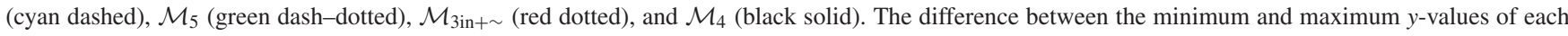
panel is kept constant to better compare the variability in $\log (p(\boldsymbol{d} \mid \mathcal{M}))$ for each model we considered.

However, the chance of sampling from the posterior mode will decrease with increasing dimensionality, which could ultimately lead to an estimate of $\widehat{p(\boldsymbol{d} \mid \mathcal{M})}$ that is not efficient.

One way around this is to sample from $g(\boldsymbol{\theta})$ within some truncated subspace, $\mathcal{T}$. This new distribution $g_{\mathcal{T}}(\boldsymbol{\theta})$ is proportional to $g(\boldsymbol{\theta})$ inside $\mathcal{T}$ and renormalized to be a proper probability density. Equation (5) can be rewritten as

$f \times \widehat{p(\boldsymbol{d} \mid \mathcal{M}}) \approx \frac{1}{N} \sum_{\boldsymbol{\theta}_{i} \sim g_{\mathcal{T}}(\boldsymbol{\theta})} \frac{p\left(\boldsymbol{d} \mid \boldsymbol{\theta}_{i}, \mathcal{M}\right) p\left(\boldsymbol{\theta}_{i} \mid \mathcal{M}\right)}{g_{\mathcal{T}}\left(\boldsymbol{\theta}_{i}\right)}$,

where $f$ is a factor that specifies what fraction of $p(\boldsymbol{d} \mid \boldsymbol{\theta}, \mathcal{M}) p\left(\boldsymbol{\theta}_{i} \mid \mathcal{M}\right)$ lies within $\mathcal{T}$. We can estimate $f$ with an MCMC sample. By counting what fraction of our posterior samples fell within $\mathcal{T}, f_{\text {MCMC }}$, we can rearrange equation (6) to give us $\widehat{p(\boldsymbol{d} \mid \mathcal{M})}$ :

$\widehat{p(\boldsymbol{d} \mid \mathcal{M})} \approx \frac{1}{N \times f_{\mathrm{MCMC}}} \sum_{\boldsymbol{\theta}_{i} \sim g_{\mathcal{T}}(\boldsymbol{\theta})} \frac{p\left(\boldsymbol{d} \mid \boldsymbol{\theta}_{i}, \mathcal{M}\right) p\left(\boldsymbol{\theta}_{i} \mid \mathcal{M}\right)}{g_{\mathcal{T}}\left(\boldsymbol{\theta}_{i}\right)}$.

Guo (2012) and Weinberg, Yoon \& Katz (2013) provide more detailed prescriptions and investigations of this method.

There are two competing effects when choosing the size of our subspace $\mathcal{T}$. If $\mathcal{T}$ is large (i.e. occupies nearly all of the posterior distribution), then $f_{\mathrm{MCMC}}$ approaches 1 and we return to our basic importance sampling algorithm. If $\mathcal{T}$ occupies a much smaller region, then we are more likely to sample from near the posterior mode, but $f_{\mathrm{MCMC}}$ approaches 0 , making it difficult to accurately estimate $\widehat{p(\boldsymbol{d} \mid \mathcal{M})}$. We must carefully choose a $\mathcal{T}$ that will provide a robust estimate of $\widehat{p(\boldsymbol{d} \mid \mathcal{M})}$. Guo (2012) found that for a three- and four-planet system, truncating the posterior distribution from $-2 \sigma$ to $+2 \sigma$ was roughly optimal, where $\sigma$ is the standard deviation of each respective model parameter. We tested several subspace sizes and found that $\pm 1.5 \sigma$ worked well for our problem.
To draw from this distribution, we create a vector $z$ whose components are independent draws from a standard normal $\mathcal{N}(0,1)$ truncated at $-1.5 \sigma$ and $+1.5 \sigma$ for each of our vector components. Each $\boldsymbol{\theta}_{i}$ is generated as $\boldsymbol{\mu}+\boldsymbol{A} \boldsymbol{z}$, where $\boldsymbol{A}$ is the Cholesky decomposition of $\boldsymbol{\Sigma}$. We generate $10^{7}$ samples from $g(\boldsymbol{\theta})$, which provided a robust estimate of $p(\boldsymbol{d} \mid \mathcal{M})$ for each of our five competing models described in Section 4. Fig. 2 shows how well the importance sampling algorithm converged for each model.

\section{COPLANAR MODELS}

Before attempting to relax the coplanarity constraint, we wish to assess the evidence for all the GJ 876 planets, $e$ in particular.

We apply RUN DMC to our cumulative set of 367 RV observations using a coplanar model but allowing for a systematic orbital inclination. In particular, we consider five different models: one with just the outermost three planets $\left(\mathcal{M}_{\text {3out }}\right)$, one with just the innermost three planets $\left(\mathcal{M}_{3 \text { in }}\right)$, one with the innermost three planets plus an $\sim 124 \mathrm{~d}$ sinusoid to mimic either a fourth planet on a circular orbit or a naive model for a stellar activity signal $\left(\mathcal{M}_{3 \text { in }+\sim}\right)$, one with all four planets $\left(\mathcal{M}_{4}\right)$, and one with a putative fifth planet $\left(\mathcal{M}_{5}\right)$.

We calculate five fully marginalized likelihoods (four Bayes factors) and summarize the results in Table 2. Our methodology for computing these probabilities is described in Section 3.4.

The Bayes factor for $\mathcal{M}_{3 \text { in }} / \mathcal{M}_{3 \text { out }}$ is $\sim 10^{31}$, decisively favouring $\mathcal{M}_{3 \text { in }}$. This was expected since $\mathcal{M}_{3 \text { in }}$ accounts for the three most significant RV signals in GJ 876. The Bayes factor for $\mathcal{M}_{3 \text { in }+\sim} / \mathcal{M}_{3 \text { in }}$ is $\sim 10^{9}$. Despite the increased parametrization of $\mathcal{M}_{3 \text { in }+\sim}$, this model with four signals is decisively favoured over $\mathcal{M}_{3 \text { in }}$. The Bayes factor for $\mathcal{M}_{4} / \mathcal{M}_{3 \text { in }+\sim}$ is $\sim 30$. The ratio of the evidence for a fully interacting four-planet model $\mathcal{M}_{4}$ relative to an interacting threeplanet model with a decoupled $\sim 124 \mathrm{~d}$ signal $\mathcal{M}_{3 \text { in }+\sim \text { is modest. }}$ 
Table 2. $\chi_{\text {eff }}^{2}$, $\log$ evidence $[\log (p(\boldsymbol{d} \mid \mathcal{M})]$, and Bayes factors computed for comparison of a finite set of models. The Bayes factors in rightmost column in this table correspond to the ratio of adjacent values of $p(\boldsymbol{d} \mid \mathcal{M})$ in the column immediately to the left.

\begin{tabular}{lcccc}
\hline Model & Description & $\chi_{\text {eff }}^{2}$ & $\log (p(\boldsymbol{d} \mid \mathcal{M}))$ & Bayes factor \\
\hline $\mathcal{M}_{5}$ & $\mathcal{M}_{4}+$ injected fifth & $743.8 \pm_{9.5}^{11.8}$ & -1255.159 & $\sim 10^{-3}$ \\
$\mathcal{M}_{4}$ & All four planets & $768.3 \pm_{8.0}^{9.7}$ & -1248.139 & $\sim 30$ \\
$\mathcal{M}_{3 \text { in }+\sim}$ & $\mathcal{M}_{3 \text { in }}+\sim 120$ d sinusoid & $779.7 \pm_{7.5}^{8.7}$ & -1251.671 & $\sim 10^{9}$ \\
$\mathcal{M}_{3 \text { in }}$ & Three innermost planets & $877.4 \pm_{6.9}^{8.3}$ & -1273.519 & $\sim 10^{31}$ \\
$\mathcal{M}_{\text {3out }}$ & Three outermost planets & $1045.7 \pm_{7.9}^{8.7}$ & -1345.970 & \\
\hline
\end{tabular}

Regardless, a model with four signals is overwhelmingly preferred over a model with three signals.

Strictly considering the results above, we would not be able to select $\mathcal{M}_{4}$ over $\mathcal{M}_{3 \text { in }+\sim}$. Decisive model selection requires at least a few orders of magnitude in separation of $p(\vec{d} \mid \mathcal{M})$ for competing models. Nevertheless, we do not expect this $\sim 124 \mathrm{~d}$ candidate to be due to (an alias of) stellar magnetic activity. Rivera et al. (2010) found that after fitting for the inner three planets to the Carnegie RVs alone, the residual periodogram strongly peaked at the planet $e$ 's period. The periodogram did not contain a strong signal near the stellar rotation period (see Section 3.3), so we do not suspect that the $124 \mathrm{~d}$ signal is an alias.

For $\mathcal{M}_{5}$, we initialized RUN DMC simulations with a hypothetical planet $f$ with the following properties: $P_{f}=15.04 \mathrm{~d}, K_{f}=3 \mathrm{~m} \mathrm{~s}^{-1}$, $e_{f}=0.007, \omega_{f}=78.3$, and $M_{f}=159.8$. This set of initial conditions was inspired by an additional candidate signal uncovered by Jenkins et al. (2014) using a global periodogram method and RVs extracted from HARPS spectra using the HARPS-TERRA pipeline (Anglada-Escudé \& Butler 2012). One significant difference is that we use an RV signal much closer to the noise level and HARPS RVs based on Correia et al. (2010). However, this periodicity is suspect since it is close to an integer fraction of the two most prominent signals in the system, $c\left(P_{c} / 2\right)$ and $b\left(P_{b} / 4\right)$. This makes our choice of $\mathcal{M}_{5}$ a good test for model comparison, since the strength of this signal competes with the extra parametrization needed to model it. After burning-in, we obtain the following estimates for $\mathcal{M}_{5}$ : $P_{f}=15.07 \pm_{0.29}^{0.32} \mathrm{~d}, K_{f}=0.42 \pm_{0.31}^{0.42} \mathrm{~m} \mathrm{~s}^{-1}$, and $e_{f}=0.16 \pm_{0.12}^{0.21}$. Due to the low RV amplitude, $\omega_{f}$ and $M_{f}$ took on any value from $0^{\circ}$ to $360^{\circ}$ almost uniformly. The Bayes factor for $\mathcal{M}_{5} / \mathcal{M}_{4}$ is $\sim 10^{-3}$.

We randomly draw 10000 posterior samples from our Markov chains for each of these cases and report our values of $\chi_{\mathrm{eff}}^{2}$ in
Table 2, along with the results of our Bayesian model comparison tests. These $\chi_{\text {eff }}^{2}$ values incorporate a penalty term dependent on RV jitter (equation 5 of Nelson et al. 2014a).

We test the coplanar models for short-term orbital stability using the hybrid integrator of MERCURY (Chambers 1999). A subsample of 1000 models is integrated for $10^{6}$ years. If at any point a planet collides with another body or the semimajor axis of any planet changes by more than 50 per cent of its original value (i.e. $\left.\left|\left[a_{\text {final }}-a_{\text {initial }}\right] / a_{\text {initial }}\right|>0.5\right)$, then the simulation stops and is tagged as being unstable. This is the default set-up for our dynamical integrations unless stated otherwise.

For $\mathcal{M}_{3 \text { out }}$, we find that 93 per cent of the posterior samples are stable for the duration of the integration. The mode of instability for the remaining 7 per cent was $|\triangle a / a|$ of planet $e$ exceeding 0.5 . This typically happened when $e_{e}>0.14$. For both $\mathcal{M}_{3 \text { in }}$ and $\mathcal{M}_{3 \text { in }+\sim, \text { all }}$ of the posterior samples are stable for the same integration timespan. For $\mathcal{M}_{4}, 99$ per cent of the posterior samples are stable. For $\mathcal{M}_{5}$, only 18 per cent of the posterior samples were stable. 77 per cent of models had $|\triangle a / a|$ of $e$ exceeding 0.5 , and at least 5 per cent of models involve a planetary collision. Some simulations had both instabilities occur.

In $\mathcal{M}_{4}$, both the inner and outer Laplace pair were locked in a 2:1 MMR, i.e. at least one resonant argument was librating (for details on resonant arguments, see Section 6), for nearly all of our posterior samples. The Laplace argument was also librating in nearly all of our samples.

Based on these previous works and the results of Sections 3.3 and 3.4, we conclude that $\mathcal{M}_{4}$ is the best model to generate these observations to date. With a four-planet model decisively chosen, we report estimates of orbital parameters and instrumental properties based on a coplanar model in Tables 3 and 4, respectively.

Table 3. Estimates of the osculating orbital elements for all the known GJ 876 planets from self-consistent, coplanar dynamical fits. Estimates are computed using 15.9, 50, and 84.1 percentiles. For other details, refer to Section 4.

\begin{tabular}{|c|c|c|c|c|}
\hline Parameter & Planet $d$ & Planet $c$ & Planet $b$ & Planet $e$ \\
\hline$K\left(\mathrm{~m} \mathrm{~s}^{-1}\right)$ & $6.01 \pm 0.30$ & $87.93 \pm 0.36$ & $213.94 \pm_{0.38}^{0.37}$ & $3.31 \pm_{0.42}^{0.38}$ \\
\hline$a(\mathrm{au})$ & $0.02183911 \pm_{0.00000018}^{0.00000019}$ & $0.135989 \pm_{0.000021}^{0.000023}$ & $0.218585 \pm 0.000026$ & $0.3514 \pm 0.0025$ \\
\hline$e$ & $0.119 \pm_{0.052}^{0.050}$ & $0.2531 \pm 0.0031$ & $0.0368 \pm_{0.0025}^{0.0019}$ & $0.031 \pm_{0.025}^{0.030}$ \\
\hline$i\left(^{\circ}\right)$ & \multicolumn{4}{|c|}{$53.19 \pm_{1.43}^{1.39}$} \\
\hline$\omega\left(^{\circ}\right)$ & $-140.90 \pm_{23.85}^{286.32}$ & $115.96 \pm_{1.35}^{1.21}$ & $110.80 \pm_{3.91}^{3.86}$ & $129.56 \pm_{185.78}^{36.31}$ \\
\hline$M\left({ }^{\circ}\right)$ & $301.87 \pm_{286.24}^{24.82}$ & $-220.68 \pm_{1.35}^{1.38}$ & $-285.38 \pm_{4.11}^{4.15}$ & $-175.89 \pm_{41.44}^{190.80}$ \\
\hline$\omega+M\left({ }^{\circ}\right)$ & $161.71 \pm_{6.71}^{6.43}$ & $-104.74 \pm_{0.64}^{0.63}$ & $-174.62 \pm_{0.45}^{0.56}$ & $-45.05 \pm_{10.97}^{9.73}$ \\
\hline
\end{tabular}


Table 4. Systematic offset and jitter estimates based on a coplanar orbital model. Estimates are computed using 15.9, 50, and 84.1 percentiles. For other details, refer to Section 3.2.

\begin{tabular}{lcc}
\hline Data set & Offset $\left(\mathrm{m} \mathrm{s}^{-1}\right)$ & Jitter $\left(\mathrm{m} \mathrm{s}^{-1}\right)$ \\
\hline HIRES Carnegie (pre-upgrade) & $50.48 \pm_{0.33}^{0.34}$ & $2.38 \pm_{0.23}^{0.25}$ \\
HIRES Carnegie (post-upgrade) & $52.20 \pm_{0.57}^{0.56}$ & \\
HIRES California (post-upgrade) & $8.18 \pm 0.94$ & $6.82 \pm_{0.63}^{0.74}$ \\
ELODIE & $-1903.81 \pm_{4.76}^{4.48}$ & $18.56 \pm_{3.94}^{4.73}$ \\
CORALIE & $-1864.40 \pm_{3.72}^{3.76}$ & $20.36 \pm_{2.84}^{3.30}$ \\
HARPS & $-1337.64 \pm_{0.43}^{0.44}$ & $1.63 \pm_{0.24}^{0.26}$ \\
\hline
\end{tabular}

Before moving on to our 3D model, we consider the significance of the relativistic precession rate $\dot{\omega}_{\text {rel }}$ for planet $d$, since it is estimated to have a moderate eccentricity $\left(e_{d} \sim 0.1\right)$ and is close to its host star. While the rapid resonant interactions amongst the outer three planets dominate their orbital evolution, the innermost planet is mostly dynamically decoupled and undergoes secular perturbations. A value of $\dot{\omega}_{\text {rel }}$ comparable to the secular precession rate $\dot{\omega}_{\text {sec }}$ tends to aggravate an instability (Ford et al. 2000), while an $\dot{\omega}_{\text {rel }}$ that is much more rapid than $\dot{\omega}_{\mathrm{sec}}$ could quench secular effects (Adams \& Laughlin 2006a,b).

We approximate the precession rate

$\dot{\omega}_{\text {rel }} \approx \frac{7.78}{1-e_{d}^{2}}\left(\frac{M_{\star}}{\mathrm{M}_{\odot}}\right)\left(\frac{a_{d}}{0.05 \mathrm{au}}\right)^{-1}\left(\frac{P_{d}}{1 \mathrm{~d}}\right)^{-1} \frac{\text { degrees }}{\text { century }}$,

where the subscript $d$ refers to planet $d$ and each parameter is measured in the units of their respective normalization (Jordán \& Bakos 2008). We evaluate equation (8) for 1000 four-planet models described and find $\dot{\omega}_{\text {rel }}=3.45 \pm_{0.03}^{0.05}$ degrees per century. Baluev (2011) found that the effects of correlated noise result in a smaller estimate of $e_{d}$, though this does not change $\dot{\omega}_{\text {rel }}$ significantly. The typical $\dot{\omega}_{\text {sec }}$ for the same models was $88 \pm 2$ degrees per century.

Ultimately, we wish to obtain a large sample of stable models but being careful not to neglect any mechanisms that could potentially stabilize a significant fraction of our models. Therefore, relativistic precession will not be included in our final long-term integrations.

\section{RESULTS FOR 3D MODEL + LONG-TERM ORBITAL STABILITY}

The planet-planet interactions in GJ 876 are so strong that the physical planet masses must be used in the modelling process. Next, we allow RUN DMC to consider the full range of parameters associated with a 3D orbit for a four-planet model. To provide a frame of reference, we set $\Omega_{d}=0^{\circ}$. For our set of initial conditions for RUN DMC, we start with our posterior samples from $\mathcal{M}_{4}$ and perturb each inclination and ascending node value (excluding $\Omega_{d}$ ) by adding a number drawn randomly and uniformly between $-1^{\circ}$ and $+1^{\circ}$. RUN DMC burned-in for about 20000 generations until a long-term steady state was reached. By modelling these extra variables, our parameter space grows from 32 dimensions (four planets $\times$ five orbital elements $\{P, K, e, \omega, M\}+i_{\text {sys }}+$ six offsets + five jitters) to 38 .

Additionally, we can obtain even more precise estimates by constraining the $3 \mathrm{D}$ orbits of the planets from a direct analysis of the RV data and by demanding orbital stability. In the end, we report 1000 solutions where the planetary system is dynamically stable for at least $10^{7}$ years. Our full procedure is described below and condensed into Table 5.

We start by randomly drawing 10000 posterior samples from our initial 3D MCMC run (Set1) for our first set of stability tests. The model parameter estimates for these samples are shown in Tables 6 and 7. These systems are integrated for $10^{5}$ years, and our conditions for stability are identical to those mentioned in Section 4. Only about 11 per cent of our initial conditions passed our stability criterion, 48 per cent of our sample show planet $d$ falling into the central star, and 41 per cent of our sample show planet $e$ 's semimajor axis suddenly changing by $|\triangle a / a|>0.5$. We visually inspected the results and determined which model parameters were most important for distinguishing between 'stable' and 'unstable' regimes of parameter space. We find that the mutual inclination between planet pairs is the most sensitive parameter in regard to the system's orbital stability. Henceforth, mutual inclinations between adjacent pairs of planets will be labelled as such: e.g. $\Phi_{\mathrm{dc}}$ for the mutual inclination between planets $d$ and $c$ (see equation 1). For planet $d, 60^{\circ}<\Phi_{\mathrm{dc}}<120^{\circ}$ causes $d$ to undergo Kozai-like perturbations, pumping its eccentricity enough so that its periastron crosses the stellar surface (Fig. 3). Fig. 4 shows the joint parameter distribution for $\cos \left(\Phi_{\mathrm{cb}}\right)$ and $\cos \left(\Phi_{\mathrm{be}}\right)$ based on Set1 (contours). We choose this parametrization over $\Phi_{\mathrm{cb}}$ and $\Phi_{\mathrm{be}}$ to visually demonstrate how consistent our solutions are with a coplanar system. For planet $e$, all initial conditions with $\Phi_{\text {be }}>13^{\circ}\left(\cos \left(\Phi_{\text {be }}\right)<0.9781\right)$ led to a sudden and large change in planet $e$ 's semimajor axis. Therefore, we perform a new set of RUN DMC simulations in which we restrict the parameter space to exclude the unstable mutual inclinations found above, i.e. $60^{\circ}<\Phi_{\mathrm{dc}}<120^{\circ}, \Phi_{\mathrm{be}}>13^{\circ}$. Although we see some large values of $\Phi_{\mathrm{cb}}$ correspond to an instability, we do not restrict the range of $\Phi_{\mathrm{cb}}$ for analysis in Set 2. RUN DMC is applied again on the same RV data set, but now the resulting posterior sample (Set 2 ) is not as heavily diluted by wildly unstable models.

We repeat the above procedure one more time, integrating the Set 2 for $10^{6}$ years. We find that the initial conditions are unstable, unless $0^{\circ}<\Phi_{\mathrm{cb}}<4^{\circ}$ and $0^{\circ}<\Phi_{\mathrm{be}}<3 \sqrt{16-\Phi_{\mathrm{cb}}^{2}}$. We run RUN DMC once more while implementing these tighter constraints to obtain a new set of posterior samples for Set3, which are subsequently integrated for $10^{7}$ years. Parameter estimates of 1000 stable

Table 5. The different orbital parameter constraints applied to RUN DMC. Here we show what range of parameter space RUN DMC is allowed to explore for each set of simulations. The initial ensemble for a particular set is generated based on a stable regime found from the previous set.

\begin{tabular}{|c|c|c|c|c|}
\hline Sample set & & Constraints during RUN & & Then integrate for... \\
\hline Set1 & $0^{\circ}<\Phi_{\mathrm{dc}}<180^{\circ}$ & $0^{\circ}<\Phi_{\mathrm{cb}}<180^{\circ}$ & $0^{\circ}<\Phi_{\mathrm{be}}<180^{\circ}$ & $10^{5} \mathrm{yr}$ \\
\hline Set 2 & $\begin{aligned} 0^{\circ} & <\Phi_{\mathrm{dc}}<60^{\circ} \\
120^{\circ} & <\Phi_{\mathrm{dc}}<180^{\circ}\end{aligned}$ & $0^{\circ}<\Phi_{\mathrm{cb}}<180^{\circ}$ & $0^{\circ}<\Phi_{\mathrm{be}}<13^{\circ}$ & $10^{6} \mathrm{yr}$ \\
\hline Set3 & $\begin{aligned} 0^{\circ} & <\Phi_{\mathrm{dc}}<60^{\circ} \\
120^{\circ} & <\Phi_{\mathrm{dc}}<180^{\circ}\end{aligned}$ & $0^{\circ}<\Phi_{\mathrm{cb}}<4^{\circ}$ & $0^{\circ}<\Phi_{\mathrm{be}}<3 \sqrt{16-\Phi_{\mathrm{cb}}^{2}}$ & $10^{7} \mathrm{yr}$ \\
\hline
\end{tabular}


Table 6. Estimates of the osculating orbital elements for all the known GJ 876 planets from self-consistent, 3D dynamical fits. The top value in each cell is estimated directly from the RVs, and the bottom value imposes dynamical stability for $10^{7}$ years. Estimates are computed using 15.9, 50, and 84.1 percentiles. Upper limits on $\Phi$ are based on a 99 per cent credible interval. For other details, refer to Section 5 .

\begin{tabular}{|c|c|c|c|c|}
\hline Parameter & Planet $d$ & Planet $c$ & Planet $b$ & Planet $e$ \\
\hline$P(\mathrm{~d})$ & $\begin{array}{l}1.937891 \pm_{0.000034}^{0.000037} \\
1.937870 \pm_{0.000028}^{0.00025}\end{array}$ & $\begin{array}{l}30.0758 \pm_{0.0078}^{0.0084} \\
30.0766 \pm_{0.0065}^{0.0073}\end{array}$ & $\begin{array}{c}61.094 \pm 0.013 \\
61.087 \pm_{0.012}^{0.011}\end{array}$ & $\begin{array}{l}123.50 \pm_{1.33}^{1.65} \\
124.72 \pm_{1.41}^{1.26}\end{array}$ \\
\hline$K\left(\mathrm{~m} \mathrm{~s}^{-1}\right)$ & $\begin{array}{l}6.13 \pm_{0.35}^{0.39} \\
6.11 \pm_{0.40}^{0.35}\end{array}$ & $\begin{array}{l}88.74 \pm_{0.62}^{0.56} \\
88.33 \pm_{0.44}^{0.47}\end{array}$ & $\begin{array}{l}213.63 \pm_{0.48}^{0.50} \\
213.71 \pm_{0.41}^{0.43}\end{array}$ & $\begin{array}{l}3.18 \pm_{0.40}^{0.51} \\
3.44 \pm_{0.41}^{0.42}\end{array}$ \\
\hline $0.37 \mathrm{M} \odot$ & $7.03 \pm_{0.90}^{3.55}$ & $274.2 \pm_{10.0}^{10.1}$ & $850.5 \pm_{16.0}^{16.6}$ & $15.45 \pm_{2.32}^{2.99}$ \\
\hline$m-\frac{M_{\star}}{M^{\prime}}\left(\mathbf{I}_{\oplus}\right)$ & $6.90 \pm_{0.81}^{3.40}$ & $267.9 \pm_{5.9}^{6.1}$ & $848.5 \pm_{17.1}^{16.4}$ & $17.16 \pm_{1.96}^{2.18}$ \\
\hline$a(\mathrm{au})$ & $\begin{array}{l}0.02183945 \pm_{0.00000026}^{0.00000034} \\
0.02183930 \pm_{0.00000022}^{0.00000022}\end{array}$ & $\begin{array}{l}0.135984 \pm_{0.000023}^{0.000026} \\
0.135985 \pm_{0.000019}^{0.00022}\end{array}$ & $\begin{array}{l}0.218609 \pm_{0.000031}^{0.000034} \\
0.218589 \pm_{0.000030}^{0.000026}\end{array}$ & $\begin{array}{l}0.3495 \pm_{0.0025}^{0.0031} \\
0.3518 \pm_{0.0026}^{0.0024}\end{array}$ \\
\hline$e$ & $\begin{array}{l}0.113 \pm_{0.050}^{0.053} \\
0.108 \pm_{0.047}^{0.050}\end{array}$ & $\begin{array}{l}0.2532 \pm_{0.0031}^{0.0032} \\
0.2539 \pm_{0.0034}^{0.0032}\end{array}$ & $\begin{array}{l}0.0371 \pm_{0.0029}^{0.0019} \\
0.0365 \pm_{0.0030}^{0.0019}\end{array}$ & $\begin{array}{l}0.046 \pm_{0.030}^{0.041} \\
0.031 \pm_{0.026}^{0.027}\end{array}$ \\
\hline$e \sin \omega$ & $\begin{array}{l}-0.038 \pm_{0.058}^{0.049} \\
-0.034 \pm_{0.055}^{0.049}\end{array}$ & $\begin{array}{l}0.2208 \pm_{0.0072}^{0.0087} \\
0.2259 \pm_{0.0050}^{0.0052}\end{array}$ & $\begin{array}{l}0.0345 \pm_{0.0034}^{0.0021} \\
0.0340 \pm_{0.0038}^{0.0022}\end{array}$ & $\begin{array}{l}0.019 \pm_{0.028}^{0.027} \\
0.004 \pm_{0.013}^{0.014}\end{array}$ \\
\hline$e \cos \omega$ & $\begin{array}{l}-0.090 \pm_{0.051}^{0.049} \\
-0.088 \pm_{0.046}^{0.049}\end{array}$ & $\begin{array}{l}-0.1236 \pm_{0.0101}^{0.0135} \\
-0.1160 \pm_{0.0065}^{0.0073}\end{array}$ & $\begin{array}{l}-0.0137 \pm_{0.0021}^{0.0024} \\
-0.0137 \pm_{0.0021}^{0.0022}\end{array}$ & $\begin{array}{l}-0.025 \pm_{0.040}^{0.033} \\
-0.006 \pm_{0.038}^{0.020}\end{array}$ \\
\hline$i\left(^{\circ}\right)$ & $\begin{array}{l}87.44 \pm_{40.94}^{41.97} \\
88.26 \pm_{40.80}^{38.90}\end{array}$ & $\begin{array}{l}51.63 \pm_{2.32}^{2.64} \\
53.06 \pm_{1.72}^{1.77}\end{array}$ & $\begin{array}{l}52.61 \pm_{1.36}^{1.44} \\
52.82 \pm_{1.44}^{1.54}\end{array}$ & $\begin{array}{l}55.51 \pm_{5.89}^{7.34} \\
53.29 \pm_{3.17}^{3.16}\end{array}$ \\
\hline$\omega\left(^{\circ}\right)$ & $\begin{array}{c}-139.25 \pm_{26.97}^{288.00} \\
162.52 \pm_{6.74}^{6.67}\end{array}$ & $\begin{array}{l}119.26 \pm_{3.58}^{2.73} \\
117.12 \pm_{1.74}^{1.70}\end{array}$ & $\begin{array}{l}111.81 \pm_{4.13}^{4.57} \\
112.27 \pm_{4.13}^{4.51}\end{array}$ & $\begin{array}{c}122.34 \pm_{207.55}^{33.00} \\
-54.2 \pm_{24.0}^{23.7}\end{array}$ \\
\hline$M\left({ }^{\circ}\right)$ & $\begin{array}{l}301.24 \pm_{289.02}^{27.44} \\
300.58 \pm_{294.52}^{25.88}\end{array}$ & $\begin{array}{l}-223.46 \pm_{2.78}^{3.17} \\
-221.76 \pm_{1.72}^{2.00}\end{array}$ & $\begin{array}{l}-286.01 \pm_{5.16}^{4.30} \\
-286.82 \pm_{4.85}^{4.24}\end{array}$ & $\begin{array}{l}-184.28 \pm_{38.26}^{231.29} \\
-92.97 \pm_{119.00}^{154.83}\end{array}$ \\
\hline$\omega+M\left(^{\circ}\right)$ & $\begin{array}{l}162.52 \pm_{6.74}^{6.67} \\
162.28 \pm_{7.28}^{6.77}\end{array}$ & $\begin{array}{l}-104.33 \pm_{0.71}^{0.73} \\
-104.60 \pm_{0.61}^{0.57}\end{array}$ & $\begin{array}{l}-174.26 \pm_{0.76}^{0.57} \\
-174.64 \pm_{0.45}^{0.46}\end{array}$ & $\begin{array}{l}-54.24 \pm_{23.98}^{23.68} \\
-42.46 \pm_{8.91}^{11.62}\end{array}$ \\
\hline$\Delta \Omega\left(^{\circ}\right)$ & & $\Delta \Omega_{\mathrm{cb}}=\begin{array}{l}-2.72 \pm_{1.73}^{2.02} \\
-1.29 \pm_{0.89}^{0.96}\end{array}$ & & $\Delta \Omega_{\mathrm{be}}=\begin{array}{c}-5.01 \pm_{10.91}^{15.17} \\
1.29 \pm_{4.58}^{4.00}\end{array}$ \\
\hline$\Phi\left(^{\circ}\right)$ & & $\Phi_{\mathrm{cb}}<\begin{array}{l}6.20 \\
2.60\end{array}$ & & $\Phi_{\text {be }}<\begin{array}{l}28.5 \\
7.87\end{array}$ \\
\hline
\end{tabular}

Table 7. Systematic offset and jitter estimates based on a 3D orbital model. Estimates are computed using 15.9, 50, and 84.1 percentiles. For other details, refer to Section 3.2.

\begin{tabular}{lcc}
\hline Data set & Offset $\left(\mathrm{m} \mathrm{s}^{-1}\right)$ & Jitter $\left(\mathrm{m} \mathrm{s}^{-1}\right)$ \\
\hline HIRES Carnegie (pre-upgrade) & $50.44 \pm 0.34$ & \\
& $50.49 \pm 0.31$ & $2.40 \pm_{0.24}^{0.25}$ \\
HIRES Carnegie (post-upgrade) & $52.04 \pm_{0.55}^{0.56}$ & $2.36 \pm_{0.23}^{0.24}$ \\
& $52.12 \pm_{0.56}^{0.65}$ & \\
HIRES California (post-upgrade) & $8.02 \pm 0.90$ & $6.76 \pm_{0.64}^{0.75}$ \\
& $8.05 \pm_{0.92}^{0.95}$ & $6.93 \pm_{0.64}^{0.74}$ \\
ELODIE & $-1904.25 \pm_{4.81}^{4.58}$ & $18.97 \pm_{4.04}^{4.70}$ \\
& $-1903.83 \pm_{4.88}^{4.65}$ & $18.69 \pm_{3.81}^{5.18}$ \\
CORALIE & $-1864.32 \pm_{3.60}^{3.53}$ & $19.96 \pm_{2.78}^{3.03}$ \\
& $-1864.48 \pm_{3.52}^{3.65}$ & $19.94 \pm_{2.66}^{3.19}$ \\
HARPS & $-1337.68 \pm_{0.47}^{0.50}$ & $1.52 \pm_{0.24}^{0.27}$ \\
& $-1337.58 \pm_{0.42}^{0.41}$ & $1.57 \pm_{0.22}^{0.25}$ \\
\hline
\end{tabular}

solutions from Set3 are shown as the bottom value of each cell in Table 6, as the solid green histogram in Fig. 3 and as green dots in Fig. 4.

Performing everything described above, we find that the RVs alone place an upper limit of $\Phi_{\mathrm{cb}}<6.20$ and $\Phi_{\mathrm{be}}<28.5$ based on a 99 per cent credible interval. However, if we expect the system to remain stable over the course of $10^{7}$ years, the system must be roughly coplanar: $\Phi_{\mathrm{cb}}<2.60$ and $\Phi_{\mathrm{be}}<7.87$ based on a 99 per cent credible interval.

\section{BEHAVIOUR OF RESONANT ANGLES}

\subsection{Eccentricity resonances}

With the final 3D orbital solutions from Set 3 of Section 5, we investigate the behaviour of the critical angles associated with the MMRs relevant for this system. For both our stable coplanar and 3D orbital models, we compute the root mean square of the variability in each angle $\times \sqrt{2}$. For a system undergoing small-amplitude sinusoidal libration, this is an excellent approximation for the libration amplitude. 


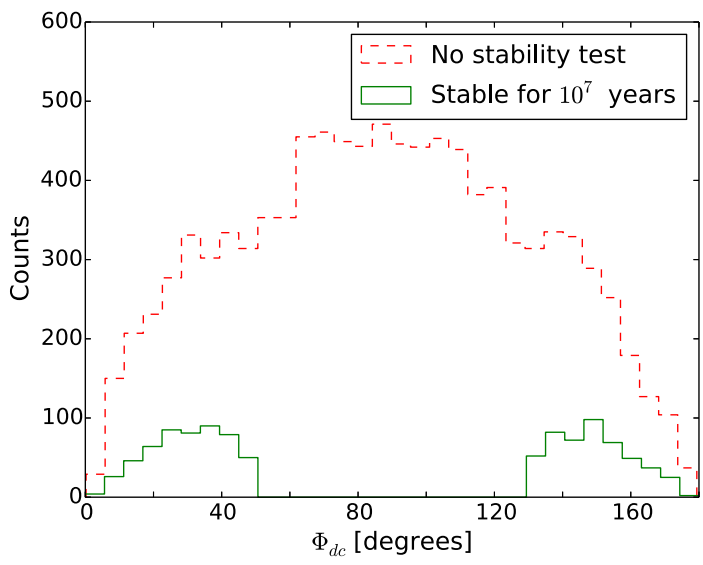

Figure 3. Mutual inclination distribution for planets $d$ and $c$. The dashed red histogram shows the initial 10000 posterior samples from Set1 before any stability tests are performed. The solid green histogram shows a sample of 1000 systems from Set3 that were stable for at least $10^{7}$ years.

For the $c$ and $b$ pair, the angles associated with the 2:1 MMR are the resonant angles, $\phi_{c}^{\mathrm{cb}}=\lambda_{c}-2 \lambda_{b}+\varpi_{c}$ and $\phi_{b}^{\mathrm{cb}}=\lambda_{c}-2 \lambda_{b}+$ $\varpi_{b}$, and the secular angle $\varpi_{c}-\varpi_{b}$, where $\lambda$ and $\varpi$ are the mean longitude and longitude of pericentre, respectively. We find that each of these angles is librating with low amplitude about $0^{\circ}$. Fig. 5 shows the distribution of the libration amplitude for angles associated with the $c$ and $b$ pair. Similarly for the $b$ and $e$ pair, the angles associated with the 2:1 MMR are the resonant angles, $\phi_{b}^{\text {be }}=\lambda_{b}-2 \lambda_{e}+\varpi_{b}$ and $\phi_{e}^{\text {be }}=\lambda_{b}-2 \lambda_{e}+\varpi_{e}$, and the secular angle $\varpi_{b}-\varpi_{e}$. We find that $\phi_{b}^{\text {be }}$ librates about $0^{\circ}$ and the other two angles are circulating. Fig. 6 shows the distribution of the libration amplitude for angles associated with the $b$ and $e$ pair. For the $c$ and $e$ pair, the angles associated with the 4:1 MMR are the resonant angles, $\phi_{0}^{\text {ce }}=\lambda_{c}-$ $4 \lambda_{e}+3 \varpi_{c}, \phi_{1}^{\mathrm{ce}}=\lambda_{c}-4 \lambda_{e}+2 \varpi_{c}+\varpi_{e}, \phi_{2}^{\mathrm{ce}}=\lambda_{c}-4 \lambda_{e}+\varpi_{c}+$
$2 \varpi_{e}$, and $\phi_{3}^{\mathrm{ce}}=\lambda_{c}-4 \lambda_{e}+3 \varpi_{e}$, and the secular angle $\varpi_{c}-\varpi_{e}$. To distinguish these angles, the subscript refers to the multiplier in front of $\varpi_{e}$. We find that four of the five angles are circulating. $\phi_{0}^{\text {ce }}$ librates about $0^{\circ}$ with low to medium amplitude. Fig. 7 shows the distribution of the libration amplitude for $\phi_{0}^{\text {ce }}$. We report all of our libration amplitudes for coplanar and dynamically stable $3 \mathrm{D}$ orbital models in Table 8 .

We find the secular angle $\omega_{b}-\omega_{e}$ is circulating, in contrast to previous studies that reported libration about $180^{\circ}$. This underscores the importance of performing self-consistent dynamical and statistical analyses when characterizing the evolution of interacting planetary systems.

The measured amplitude is interesting as it is neither so small as to imply strong dissipation nor so large as to suggest the absence of damping. Given the importance of this result, we performed additional tests to verify that our algorithm accurately characterizes the libration amplitude of the Laplace angle for both regular and chaotic systems. The methods and more detailed results of these test are presented in Appendix A.

Upon the discovery of the outermost planet, Rivera et al. (2010) found that their best-fitting systems exhibit a 4:2:1 Laplace resonance. The associated angle, $\phi_{\text {Laplace }}=\lambda_{c}-3 \lambda_{b}+2 \lambda_{e}$, evolves chaotically. Based on the Carnegie RVs alone, they found that $\phi_{\text {Laplace }}$ librates about $0^{\circ}$ with an amplitude of $40^{\circ} \pm 13^{\circ}$ for a coplanar four-planet model. Fig. 8 shows our results for the posterior distribution for the libration amplitude for the Laplace argument for both the coplanar $\left(33 \pm_{9.3}^{12.4 \circ}\right)$ and $3 \mathrm{D}\left(50.7 \pm_{10}^{7.9 \circ}\right)$ cases. The measured amplitude is interesting as it is neither so small as to imply strong dissipation nor so large as to suggest the absence of damping. Given the importance of this result, we performed additional tests to verify that our algorithm accurately characterizes the libration amplitude of the Laplace angle for both regular and chaotic systems. The methods and more detailed results of these tests are presented in Appendix A.

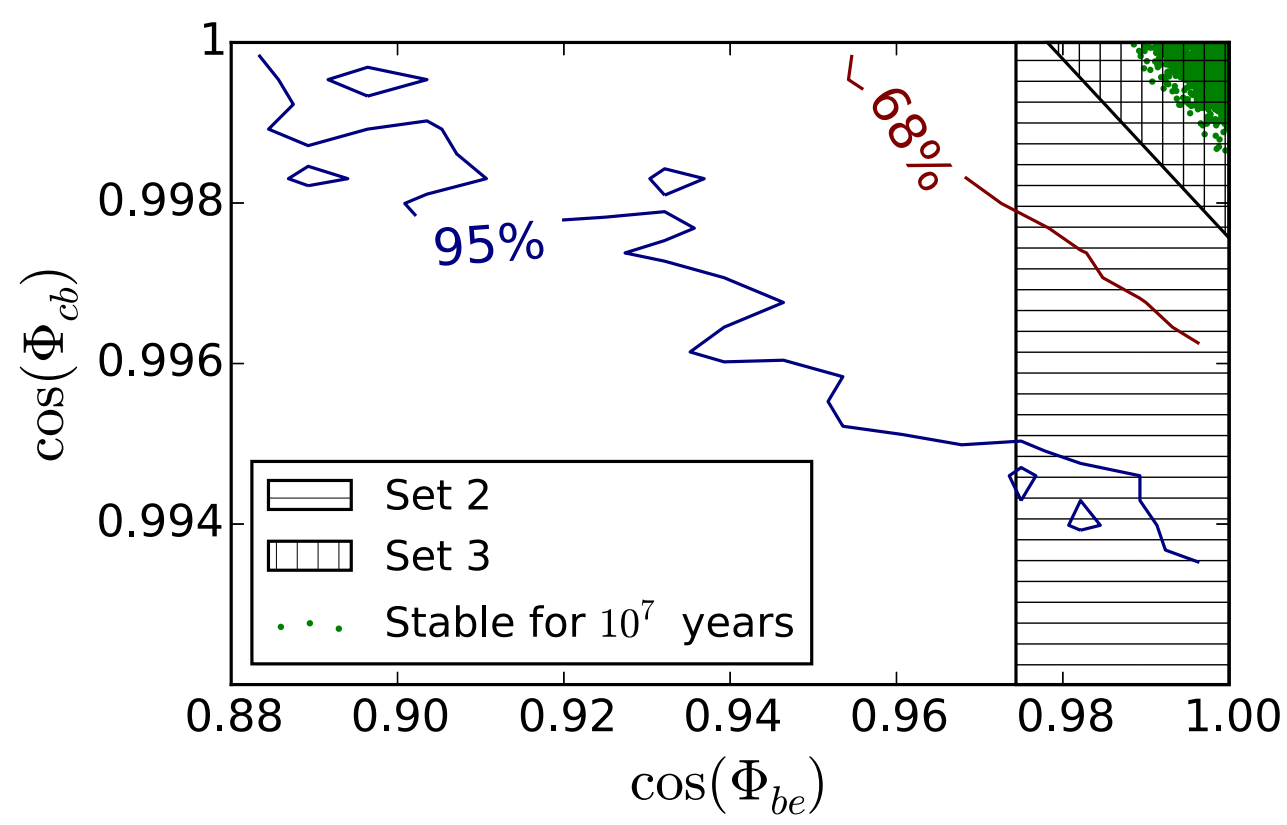

Figure 4. The joint cosine mutual inclination distribution for planets $c$ and $b$ (vertical axis) and $b$ and $e$ (horizontal axis). Contours map the approximate $1 \sigma$ (68 per cent) and $2 \sigma$ (95 per cent) credible regions for the initial 10000 posterior samples from Set 1 before any stability tests are performed. Horizontal hatch marks indicate the region for obtaining Set 2 and the vertical hatch marks indicate the region for obtaining Set 3, both described in Section 5 and Table 5 . Green dots show a sample of 1000 systems from Set 3 that were stable for at least $10^{7}$ years. 

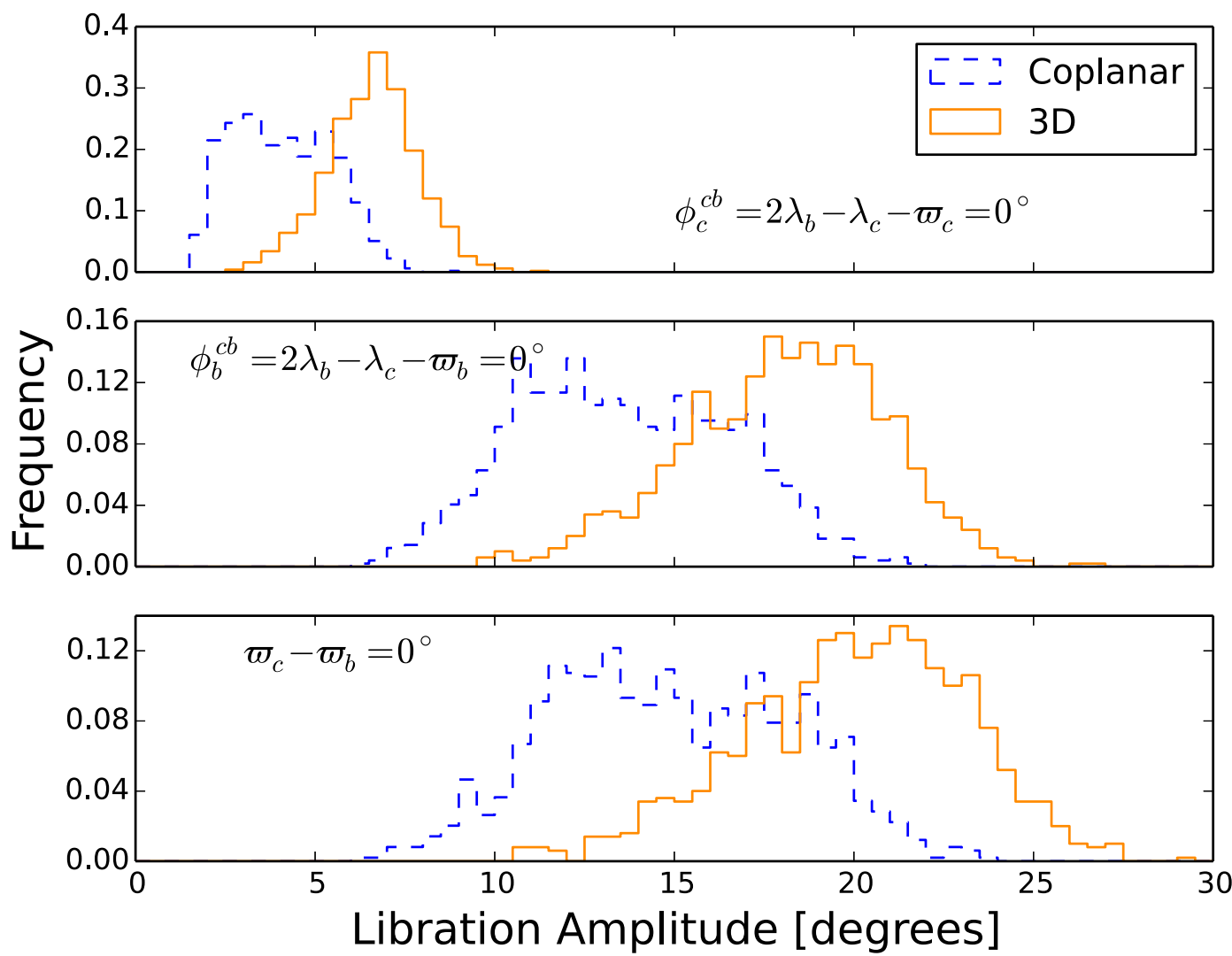

Figure 5. Libration amplitude distributions for the three resonant angles of the $c$ and $b$ planet pair in a 2:1 MMR. We compare libration amplitudes for a coplanar (blue dashed) and 3D (orange solid) orbital model. The two angles associated with the 2:1 MMR (top and middle panels) are librating about $0^{\circ}$ with low amplitude. The secular angle (bottom panel) also librates about $0^{\circ}$ with low amplitude.

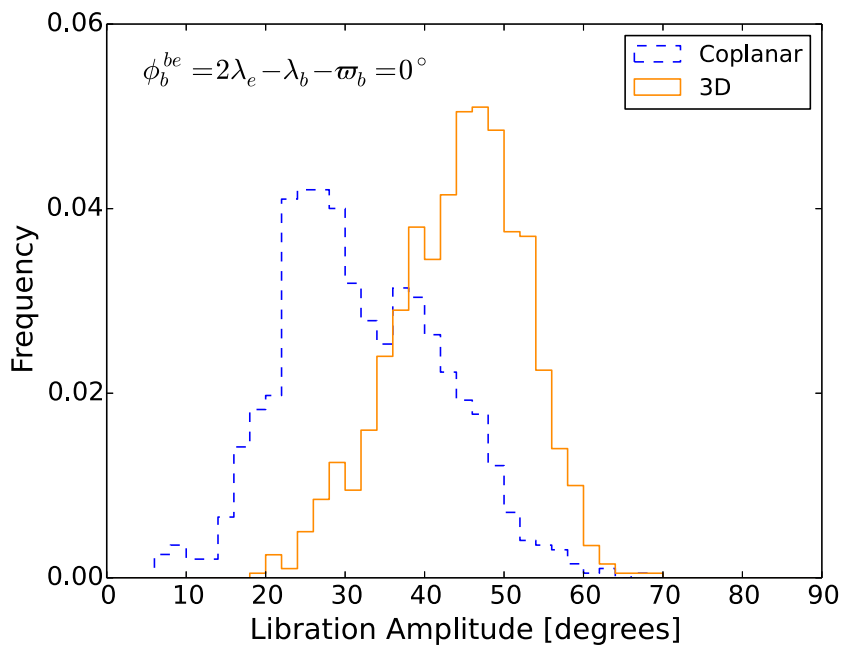

Figure 6. Libration amplitude distributions for only librating angle of the $b$ and $e$ planet pair in a 2:1 MMR. We compare libration amplitudes for a coplanar (blue dashed) and 3D (orange solid) orbital model. For the vast majority of our sets of initial conditions, the other resonant argument and the secular angle are circulating.

\subsection{Inclination resonances}

Several studies have looked at inclination excitation and inclination resonance capture during planet migration phases (Thommes \& Lissauer 2003; Lee \& Thommes 2009; Libert \& Tsiganis 2009; Teyssandier \& Terquem 2014). The general conclusion in these

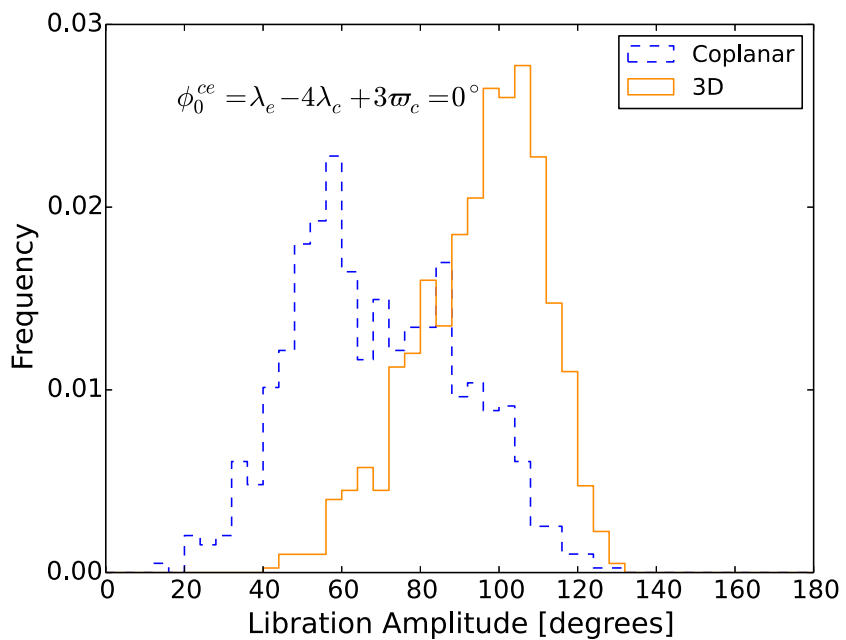

Figure 7. Libration amplitude distribution for the only librating angle of the $c$ and $e$ planet pair relative to a 4:1 MMR. We compare libration amplitudes for a coplanar (blue dashed) and 3D (orange solid) orbital model. For the vast majority of our sets of initial conditions, the three other resonant arguments and the secular angle are circulating.

studies is that inclination resonances can form during the formation phase but are likely to be short-lived. A recent study by Barnes et al. (2015) investigated the orbital evolution of MMR systems with mutually inclined orbits in the post-formation phase. They find that their synthetic systems evolve chaotically but still retain dynamical stability and remain in a MMR. Drawing upon these 
Table 8. Libration amplitudes of resonant angles. Estimates are computed using 15.9, 50, and 84.1 percentiles. See Section 6 for details.

\begin{tabular}{lcc}
\hline Angle & Amplitude, coplanar $\left(^{\circ}\right)$ & Amplitude, $3 \mathrm{D}\left({ }^{\circ}\right)$ \\
\hline$\phi_{c}^{\mathrm{cb}}$ & $4.0 \pm_{1.4}^{1.6}$ & $7.0 \pm_{1.4}^{1.3}$ \\
$\phi_{b}^{\mathrm{cb}}$ & $13.4 \pm_{1.4}^{3.0}$ & $18.69 \pm_{3.0}^{2.5}$ \\
$\varpi_{c}-\varpi_{b}$ & $14.7 \pm_{3.3}^{4.0}$ & $20.6 \pm_{3.5}^{2.8}$ \\
$\phi_{b}^{\text {be }}$ & $31.3 \pm_{8.7}^{11.9}$ & $47.9 \pm_{9.5}^{7.8}$ \\
$\phi_{e}^{\text {be }}$ & Circulating & Circulating \\
$\varpi_{b}-\varpi_{e}$ & Circulating & Circulating \\
$\phi_{0}^{\text {ce }}$ & $67.5 \pm_{19.4}^{25.4}$ & $103.2 \pm_{19.2}^{12.3}$ \\
$\phi_{1}^{\text {ce }}$ & Circulating & Circulating \\
$\phi_{2}^{\text {ce }}$ & Circulating & Circulating \\
$\phi_{3}^{\text {ce }}$ & Circulating & Circulating \\
$\varpi_{b}-\varpi_{e}$ & Circulating & Circulating \\
$\phi_{\text {Laplace }}$ & $33.0 \pm_{9.3}^{12.4}$ & $50.5 \pm_{10.0}^{7.9}$ \\
\hline
\end{tabular}

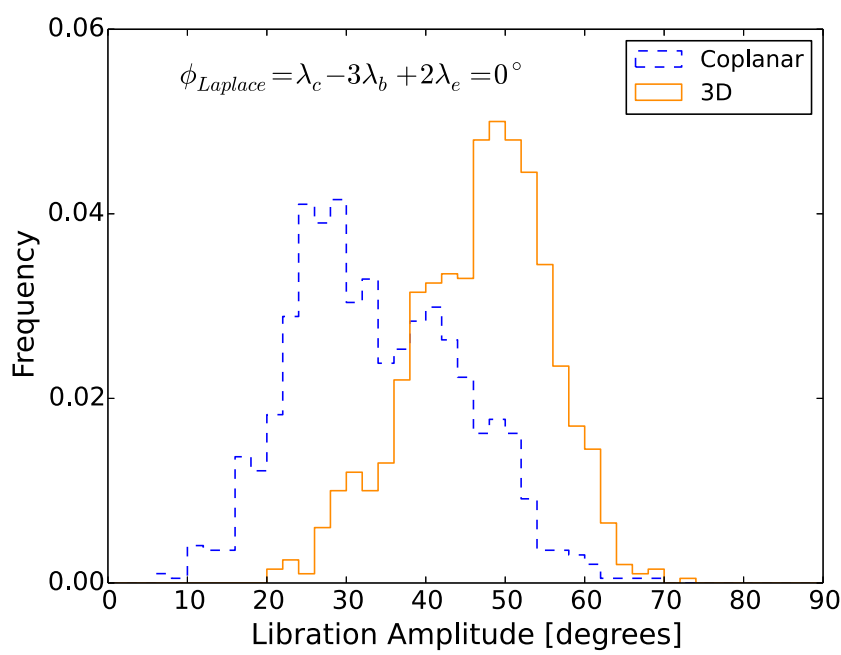

Figure 8. Libration amplitude distributions for the Laplace argument of the $c, b$, and $e$ resonant trio. We compare libration amplitudes for a coplanar (blue dashed) and 3D (orange solid) orbital model.

conclusions, they model several real RV systems near an MMR to look for similar behaviour. They find that HD 73526 (a 2:1 MMR system) and HD 60532 (a 3:1 MMR system) could be evolving chaotically given a set of initial conditions listed in their tables 9 and 10 , respectively. However, the inclination resonance arguments associated with these systems were all circulating, indicating that they were not in an inclination resonance. To date, no exoplanet system has a detected inclination resonance.

We check for an inclination resonance in GJ 876 with our dynamically stable posterior samples. First, we transform our set of initial conditions into the invariable plane,$^{2}$ then integrate these for 1000 years, logging the output every $10 \mathrm{~d}$. We compute the angles associated with the inclination resonance for the inner pair $\phi_{\text {incl }}^{\mathrm{cb}}=4 \lambda_{b}-2 \lambda_{c}-\Omega_{c}-\Omega_{b}$ and the outer pair $\phi_{\text {incl }}^{\mathrm{be}}=$ $4 \lambda_{e}-2 \lambda_{b}-\Omega_{b}-\Omega_{e}$. Visually inspecting the orbital evolution over 100 years of these models, we find that $\phi_{\text {incl }}^{\text {cb }}$ and $\phi_{\text {incl }}^{\text {be }}$ both circulate on time-scales of $\sim 5 \mathrm{yr}$. While it is possible that $\phi_{\text {incl }}$ may occasionally librate for a short period of time, this is sufficiently rare that we did not observe any such events over this baseline for all of our models.

\footnotetext{
${ }^{2}$ https://github.com/RoryBarnes/InvPlane
}

\section{SUMMARY AND DISCUSSION}

We have meaningfully constrained the 3D orbital architecture of the GJ 876 planetary system, based on 367 Doppler observations from several observing sites.

To verify the nature of the $\sim 124 \mathrm{~d}$ signal, we performed Bayesian model comparison of five different physical models, spanning three to five planets. Since each evaluation of the likelihood requires an $\mathrm{N}$ body integration for a strongly interacting planetary system like GJ 876 , Bayesian model comparison becomes computationally costly. Therefore, it was particularly important that we apply an efficient and parallelizable algorithm. We refined and applied a modified importance sampling algorithm to compute the fully marginalized likelihood, or Bayesian evidence, starting from a posterior sample computed via MCMC methods (Ford \& Gregory 2007; Guo 2012; Weinberg et al. 2013). The algorithm parallelizes readily and was implemented on GPUs using the SWARM-NG framework (Dindar et al. 2013). While previous studies have computed Bayes factors using other algorithms (e.g. nested sampling, Feroz, Hobson \& Bridges 2009; Kipping 2013; Placek, Knuth \& Angerhausen 2014; geometric-path Monte Carlo, Hou, Goodman \& Hogg 2014), these studies have assumed that the motion can be described as the linear superposition of Keplerian orbits, which is unsuitable for strongly interacting planetary systems such as GJ 876 . We believe this study to be the first example of rigorous Bayesian model comparison applied to strongly interacting planetary systems. This algorithm is relatively easy to implement and worked well even for our highdimensional ( $\sim 30-40$ parameter) models.

We determined that a four-planet model is most appropriate for the present data, based on a self-consistent Bayesian and $N$-body analysis (Sections 3.3 and 3.4). When computing the evidence for our finite set of models, we can decisively choose a model with four signals with Bayes factors exceeding $10^{3}$. We find a Bayes factor of $\sim 30$ when comparing a four-planet model $\left(\mathcal{M}_{4}\right)$ to a model with three planets plus a decoupled sinusoid, which could result from either a fourth planet in a circular orbit or a stellar activity signal $\left(\mathcal{M}_{3 \text { in }+\sim}\right)$.

On one hand, a Bayes factor of $\sim 30$ is not large enough by itself to definitively choose the four-planet model. On the other hand, we find no reason to suspect that stellar activity is masquerading as a planetary signal. Looking at activity-sensitive indicators in publicly available HARPS spectra, we measure a stellar rotation period of $95 \pm 1 \mathrm{~d}$. This does not directly correspond with any of the planets' orbital periods. As expected, the stellar rotation period and annual observing cycle lead to an alias with a frequency of $\sim 128 \mathrm{~d}$. In our coplanar model, the orbital period of $e$ is $124.5 \pm 1.3$, differing from the $128 \mathrm{~d}$ alias at slightly more than the $2 \sigma$ level. If the signal were due solely to aliasing of the annual observing cycle with the stellar rotation period, then there should be an even stronger signal corresponding to the stellar rotation in the periodogram of the RV residuals (after subtracting the RV signal due to the inner three planets). Rivera et al. (2010) and Baluev (2011) looked for but did not find this effect, concluding that the signal is best explained by planet $e$.

Through numerical integrations of systems where planet $e$ was treated as a test particle, Rivera et al. (2010) explored the parameter space near the best-fitting GJ 876 solution to the Carnegie RVs. These tests suggested that the true system might be chaotic and was likely surrounded by regions of phase space with short-lived (unstable) orbits. Martí, Giuppone \& Beaugé (2013) studied the two reported four-planet solutions to the RV data using the MEGNO method (e.g. Cincotta \& Simó 2000) and found that these trajectories 
were both chaotic and in the Laplace resonance. We show the most probable Lyapunov time to be $\sim 10 \mathrm{yr}$ for both our coplanar and dynamically stable $3 \mathrm{D}$ models (Appendix A3). This time-scale is consistent with the results of Batygin, Deck \& Holman (2015) which were based on combining a simplified dynamical model and a point estimate for orbital parameters.

Strongly interacting planetary systems like GJ 876 could have unusually large transit timing variation amplitudes, enabling detailed characterization via the transit timing technique. Upcoming missions such as TESS and PLATO would easily detect and confirm a similar system of transiting planets, but a detailed interpretation could be challenging given the anticipated observing timespans. In particular, the perturbations from GJ $876 e$ become evident only on multi-year time-scales (Libert \& Renner 2013).

While previous studies have assumed that the planets in GJ 876 follow coplanar orbits, we relax the assumption of coplanarity and allow for non-coplanar orbital configurations. We analyse the Doppler observations of GJ 876 and find that the three planets participating in the Laplace resonance must be nearly coplanar. The 99 per cent credible upper limits on the mutual inclination constrained by just the RVs are impressive for both planet pairs: $\Phi_{\mathrm{cb}}<6.20$ for the $c$ and $b$ pair and $\Phi_{\mathrm{be}}<28.5$ for the $b$ and $e$ pair. Demanding orbital stability further restricts this range providing precise constraints on the mutual inclinations: $\Phi_{\mathrm{cb}}<2.60$ and $\Phi_{\text {be }}<7$ 7.87. A plot of the posterior samples suggests that the orbits of these planets are consistent with a coplanar system (Fig. 4). Despite its rather unique orbital architecture, it seems that GJ 876 fits in with a population of $\mathrm{M}$ dwarf systems with several coplanar planets (Ballard \& Johnson 2014).

By performing the first self-consistent Bayesian analysis of the four planets in GJ 876, we are able to accurately characterize the current dynamical state of all four planets and particularly the evolution of the resonant angle associated with the Laplace resonance. We measure the amplitude of variations to be $33 \pm_{9.3}^{12.4}$ degrees for coplanar models or $50.5 \pm_{10.0}^{7.9}$ degrees for fully $3 \mathrm{D}$ models.

When measuring a positive definite quantity, observational uncertainties can bias measurements, particularly when using point estimates. For example, the best-fitting models of Doppler observations of a population of planets with nearly circular orbits will typically overestimate the planets' orbital eccentricities (Zakamska, Pan \& Ford 2011), particularly when the Doppler amplitude is only a factor of a few greater than the measurement precision. This motivated our Bayesian approach to characterizing the amplitude of variations of the Laplace angle. Additionally, we performed tests of our algorithms using simulated planetary systems that confirm our algorithm accurately characterizes the behaviour of the Laplace angle (Appendix A1). We conclude that formation theories for the GJ 876 system need to explain not only the resonant structure, but also the chaotic evolution of the Laplace angle and its sizeable amplitude.

The near integer ratio in the planets' orbital periods is not likely a result of happenstance. The probability of in situ formation yielding such a system is further reduced by the need to become trapped in a chaotic, but long-lived, multi-body resonance. Within the context of current planet formation models, the system most likely reached its current resonant configuration as the result of disc migration. Migration through a smooth gas disc would be expected to result in strong damping of eccentricities, driving the system to a state where the resonant angle librates regularly with small amplitude. Many of our simulations of a smooth migration with eccentricity damping lead to an amplitude of $\sim 1^{\circ}$, much smaller than we measure for the current system configuration.
While the observed large libration amplitude and chaotic evolution of resonant angles are contrary to the predictions of the simplest migration models, Batygin et al. (2015) recently proposed that that the observed libration of the resonant angles can be explained purely by a turbulent migration. We propose an alternative formation mechanism based on a phase of smooth disc migration which terminates abruptly (Appendix A). In our simplistic migration models, turning off the eccentricity damping impulsively caused the libration amplitude for the Laplace angle to rise from $\sim 1^{\circ}$ to $\sim$ tens of degrees. Such a scenario could arise naturally as a result of rapid dispersal of inner disc via photoevaporation.

Another potential possible formation scenario involves migration through a gas disc trapping the planets in resonance, followed by a phase of planet or planetesimal scattering. Chatterjee \& Ford (2015) showed that planetesimal scattering will naturally drive a planetary system initially in a 2:1 MMR farther apart, exciting eccentricities and perhaps even breaking the resonance.

It is possible for MMRs to arise from pure planet-planet scattering. Raymond et al. (2008) found that MMRs could form through the ejection of one planet, based on simulations starting with three planets with drastically different masses. One can distinguish between MMRs formed through scattering and migration mechanisms by the mass and orbital properties of the observed planets. Scattering usually yields planet pairs in higher order resonances (second or greater), with larger semimajor axes ( $>1 \mathrm{au}$ ) and/or with a more massive outer companion. The latter result could explain the $c$ and $b$ pair, but after considering $e$ and the observed Laplace resonance, planet-planet scattering alone becomes a less likely formation channel for the GJ 876 planets. Indeed, Moeckel \& Armitage (2012) run a set of simulations modelling multi-planet systems during disc clearing phase and subsequent gas-free (purely Newtonian) phase and find that systems were much more likely to form resonant chains if scattering events did not occur. In the presence of a planetesimal disc, already 'marginally stable' systems can also form resonant chains (Raymond, Armitage \& Gorelick 2009).

We encourage future studies to explore the predictions of these formation models for comparison to the GJ 876 system. Continued long-term RV monitoring and/or astrometric observations from Gaia could continue to improve the dynamical constraints on this landmark system.

\section{ACKNOWLEDGEMENTS}

We would like to thank our referee, Edward Thommes, for his constructive feedback that improved the manuscript. We would also like to thank Geoff Marcy and the entire of the California Planet Survey team for their long-term commitment to high-precision RVs for the GJ 876 system. BEN would like to thank Phil Gregory and Tom Loredo for useful suggestions regarding our methodology and presentation of our importance sampling algorithm. Additionally, he would also like to thank Rory Barnes and Russell Deitrick for conversations regarding chaos and inclination resonances in multiplanet systems and Roman Baluev for useful feedback regarding the significance of correlated noise.

PR acknowledges support from NSF grant AST-1126413 and the Center for Exoplanets and Habitable Worlds. MJP gratefully acknowledges the NASA Origins of Solar Systems Program grant NNX13A124G. EBF and JTW acknowledge NASA Keck PI Data Awards, administered by the NASA Exoplanet Science Institute, including awards 2007B N095Hr, 2010A N147Hr, 2011A\&B $\mathrm{N} 141 \mathrm{Hr}$, and 2012A N129Hr. This research was supported by NASA Origins of Solar Systems grant NNX09AB35G. We 
acknowledge the University of Florida High Performance Computing Center and the Pennsylvania State Research Computing and Advanced Cyberinfrastructure Group for providing computational resources and support that have contributed to the results reported within this paper. The Center for Exoplanets and Habitable Worlds is supported by the Pennsylvania State University, the Eberly College of Science, and the Pennsylvania Space Grant Consortium. We extend special thanks to those of Hawaiian ancestry on whose sacred mountain of Mauna Kea we are privileged to be guests. Without their generous hospitality, the Keck observations presented herein would not have been possible.

\section{REFERENCES}

Adams F. C., Laughlin G., 2006a, Int. J. Mod. Phys. D, 15, 2133

Adams F. C., Laughlin G., 2006b, ApJ, 649, 992

Anglada-Escudé G., Butler R. P., 2012, ApJS, 200, 15

Anglada-Escudé G., López-Morales M., Chambers J. E., 2010, ApJ, 709, 168

Ballard S., Johnson J. A., 2014, preprint (arXiv:e-prints)

Baluev R. V., 2011, Celest. Mech. Dyn. Astron., 111, 235

Barnes R., Deitrick R., Greenberg R., Quinn T. R., Raymond S. N., 2015, ApJ, 801, 101

Batygin K., Morbidelli A., 2013, AJ, 145, 1

Batygin K., Deck K. M., Holman M. J., 2015, AJ, 149, 167

Bean J. L., Seifahrt A., 2009, A\&A, 496, 249

Beaugé C., Ferraz-Mello S., Michtchenko T. A., 2003, ApJ, 593, 1124

Benedict G. F. et al., 2002, ApJ, 581, L115

Boisse I., Bouchy F., Hébrard G., Bonfils X., Santos N., Vauclair S., 2011, A\&A, 528, A4

Butler R. P., Marcy G. W., Williams E., McCarthy C., Dosanjh P., Vogt S. S., 1996, PASP, 108, 500

Chambers J. E., 1999, MNRAS, 304, 793

Chatterjee S., Ford E. B., 2015, ApJ, 803, 33

Cincotta P. M., Simó C., 2000, A\&AS, 147, 205

Correia A. C. M. et al., 2010, A\&A, 511, A21

Delfosse X., Forveille T., Mayor M., Perrier C., Naef D., Queloz D., 1998 A\&A, 338, L67

Dindar S., Ford E. B., Juric M., Yeo Y. I., Gao J., Boley A. C., Nelson B., Peters J., 2013, New Astron., 23, 6

Dumusque X., Boisse I., Santos N. C., 2014, ApJ, 796, 132

Feroz F., Hobson M. P., Bridges M., 2009, MNRAS, 398, 1601

Ford E. B., Gregory P. C., 2007, in Babu G. J., Feigelson E. D., eds, ASP Conf. Ser. Vol. 371, Statistical Challenges in Modern Astronomy IV. Astron. Soc. Pac., San Francisco, p. 189

Ford E. B., Joshi K. J., Rasio F. A., Zbarsky B., 2000, ApJ, 528, 336

Ford E. B., Moorhead A. V., Veras D., 2011, Bayesian Anal., 6, 475

France K., Linsky J. L., Tian F., Froning C. S., Roberge A., 2012, ApJ, 750, L32

France K. et al., 2013, ApJ, 763, 149

Gerlach E., Haghighipour N., 2012, Celest. Mech. Dyn. Astron., 113, 35

Goździewski K., Bois E., Maciejewski A. J., 2002, MNRAS, 332, 839

Guo P.-C., 2012, PhD thesis, Univ. Florida

Haghighipour N., Couetdic J., Varadi F., Moore W. B., 2003, ApJ, 596, 1332

Hou F., Goodman J., Hogg D. W., 2014, preprint (arXiv:e-prints)

Jenkins J. S., Yoma N. B., Rojo P., Mahu R., Wuth J., 2014, MNRAS, 441, 2253

Ji J. H., Liu L., 2006, Acta Astron. Sin., 47, 402

Ji J., Li G., Liu L., 2002, ApJ, 572, 1041

Johnson J. A. et al., 2011, AJ, 141, 16

Jones B. W., Sleep P. N., Chambers J. E., 2001, A\&A, 366, 254

Jordán A., Bakos G. Á., 2008, ApJ, 685, 543

Kammer J. A. et al., 2014, ApJ, 781, 103

Kinoshita H., Nakai H., 2001, PASJ, 53, L25

Kipping D. M., 2013, MNRAS, 434, L51

Kley W., Peitz J., Bryden G., 2004, A\&A, 414, 735
Kley W., Lee M. H., Murray N., Peale S. J., 2005, A\&A, 437, 727

Kokubo E., Yoshinaga K., Makino J., 1998, MNRAS, 297, 1067

Laughlin G., Chambers J. E., 2001, ApJ, 551, L109

Laughlin G., Butler R. P., Fischer D. A., Marcy G. W., Vogt S. S., Wolf A. S., 2005, ApJ, 622, 1182

Lee M. H., 2004, ApJ, 611, 517

Lee M. H., Peale S. J., 2002, ApJ, 567, 596

Lee M. H., Thommes E. W., 2009, ApJ, 702, 1662

Lega E., Morbidelli A., Nesvorný D., 2013, MNRAS, 431, 3494

Leinert C., Henry T., Glindemann A., McCarthy D. W., Jr, 1997, A\&A, 325, 159

Libert A.-S., Renner S., 2013, MNRAS, 430, 1369

Libert A.-S., Tsiganis K., 2009, A\&A, 493, 677

Lichtenberg A. J., Lieberman M. A., 1992, Regular and Chaotic Dynamics. Springer, Berlin

Luhman K. L., Jayawardhana R., 2002, ApJ, 566, 1132

Marcy G. W., Butler R. P., 1992, PASP, 104, 270

Marcy G. W., Butler R. P., Vogt S. S., Fischer D., Lissauer J. J., 1998, ApJ, 505, L147

Marcy G. W., Butler R. P., Fischer D., Vogt S. S., Lissauer J. J., Rivera E. J., 2001, ApJ, 556, 296

Martí J. G., Giuppone C. A., Beaugé C., 2013, MNRAS, 433, 928

Milani A., Nobili A. M., 1983, Celest. Mech., 31, 213

Moeckel N., Armitage P. J., 2012, MNRAS, 419, 366

Montet B. T., Crepp J. R., Johnson J. A., Howard A. W., Marcy G. W., 2014 ApJ, 781, 28

Murray N., Paskowitz M., Holman M., 2002, ApJ, 565, 608

Nelson B. E., Ford E. B., Payne M. J., 2014a, ApJS, 210, 11

Nelson B. E., Ford E. B., Wright J. T., Fischer D. A., von Braun K., Howard A. W., Payne M. J., Dindar S., 2014b, MNRAS, 441, 442

Patience J. et al., 2002, ApJ, 581, 654

Placek B., Knuth K. H., Angerhausen D., 2014, ApJ, 795, 112

Podlewska-Gaca E., Papaloizou J. C. B., Szuszkiewicz E., 2012, MNRAS, 421, 1736

Rauch K. P., Holman M., 1999, AJ, 117, 1087

Raymond S. N., Barnes R., Armitage P. J., Gorelick N., 2008, ApJ, 687, L107

Raymond S. N., Armitage P. J., Gorelick N., 2009, ApJ, 699, L88

Rivera E., Haghighipour N., 2007, MNRAS, 374, 599

Rivera E. J., Lissauer J. J., 2001, ApJ, 558, 392

Rivera E. J. et al., 2005, ApJ, 634, 625

Rivera E. J., Laughlin G., Butler R. P., Vogt S. S., Haghighipour N., Meschiari S., 2010, ApJ, 719, 890

Robertson P., Mahadevan S., 2014, ApJ, 793, L24

Robertson P., Mahadevan S., Endl M., Roy A., 2014, Science, 345, 440

Shankland P. D. et al., 2006, ApJ, 653, 700

Snellgrove M. D., Papaloizou J. C. B., Nelson R. P., 2001, A\&A, 374, 1092

Tan X., Payne M. J., Lee M. H., Ford E. B., Howard A. W., Johnson J. A., Marcy G. W., Wright J. T., 2013, ApJ, 777, 101

Teyssandier J., Terquem C., 2014, MNRAS, 443, 568

Thommes E. W., 2005, ApJ, 626, 1033

Thommes E. W., Lissauer J. J., 2003, ApJ, 597, 566

Thommes E. W., Matsumura S., Rasio F. A., 2008, Science, 321, 814

Valenti J. A., Butler R. P., Marcy G. W., 1995, PASP, 107, 966

van Leeuwen F. ed., 2007, Astrophysics and Space Science Library, Vol.

350, Hipparcos, the New Reduction of the Raw Data. Springer, Berlin

Veras D., 2007, Celest. Mech. Dyn. Astron., 99, 197

Veras D., Ford E. B., 2010, ApJ, 715, 803

Vogt S. S. et al., 1994, in Crawford D. L., Craine E. R., eds, Proc. SPIE, Vol. 2198, Instrumentation in Astronomy VIII. SPIE, Bellingham, p. 362

von Braun K. et al., 2014, MNRAS, 438, 2413

Weinberg M. D., Yoon I., Katz N., 2013, preprint (arXiv:e-prints)

Wisdom J., 2006, AJ, 131, 2294

Wisdom J., Holman M., 1991, AJ, 102, 1528

Wisdom J., Holman M., 1992, AJ, 104, 2022

Wisdom J., Holman M., Touma J., 1996, in Marsden J. E., Patrick G. W., Shadwick W. F., eds, Fields Institute Communications, Vol. 10, 
Integration Algorithms and Classical Mechanics. Am. Math. Soc., Providence, p. 217

Zakamska N. L., Pan M., Ford E. B., 2011, MNRAS, 410, 1895

Zechmeister M., Kürster M., 2009, A\&A, 496, 577

Zhou J.-L., Sun Y.-S., 2003, ApJ, 598, 1290

\section{SUPPORTING INFORMATION}

Additional Supporting Information may be found in the online version of this article:

\section{sample_stable.out \\ sample.out \\ newKeckVels}

(http://mnras.oxfordjournals.org/lookup/suppl/doi:10.1093/mnras/ stv2367/-/DC1).

Please note: Oxford University Press is not responsible for the content or functionality of any supporting materials supplied by the authors. Any queries (other than missing material) should be directed to the corresponding author for the article.

\section{APPENDIX A: FITTING SYNTHETIC SYSTEMS WITH KNOWN PROPERTIES}

Batygin et al. (2015) provide an elegant explanation of how such a chaotically evolving Laplace angle could have formed, and show that it can provide limits on the system's early formation: smooth migration can only form systems with relatively small libration amplitude and regular non-chaotic evolution; stochastic migration is required to form chaotically evolving systems with large libration amplitudes.

At face value, our results (Section 6) provide evidence for chaotic evolution of the Laplace argument in the GJ 876 system. Given the importance of this result, we performed additional tests to determine whether small libration amplitude is likely to be erroneously classified as chaotic orbital evolution. We simulated RV observations of a variety of synthetic coplanar systems and then applied our RUN DMC algorithm to verify that the recovered parameter estimates (in particular the libration amplitude of the Laplace angle) are consistent with their input values.

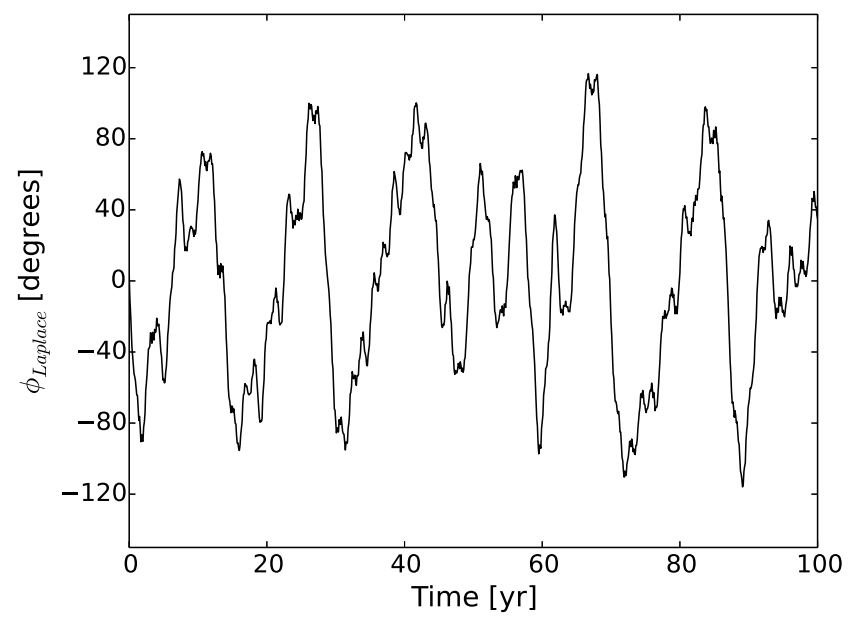

\section{A1 Methodology: creating synthetic planetary systems and} data sets

Batygin (private communication) provided orbital elements for the results of one of their stochastic migration simulations. We integrated this system forward, confirming that the evolution of the Laplace resonance angle, $\phi_{\text {Laplace }}$, is indeed chaotic and has a libration amplitude $\sim 80^{\circ}$. This synthetic system will be referred to as $\mathrm{S}_{\text {Chaos:80 }}$ henceforth (Fig. A1, left).

To complement this, we then performed a number of smooth migration simulations in which the resultant simulations are regular and have low libration amplitudes. These migration simulations used the damped $a$ and $e$ method described in Lee \& Peale (2002), which was implemented in a modified version of MERCURY (Chambers 1999). We set up three-planet systems so that each initial planetary period ratio was a little larger than 2:1 with their inner neighbour. Then, we applied the damped-migration model to the outer planet until it caught into a $2: 1$ resonance with the middle planet. We continued the forced, damped migration until this outer resonant pair caught into a 4:2:1 resonance with the innermost planet. Finally, we removed all forms of damping from the system and let them evolve for $\sim 10^{5}$ years, ensuring that they were in an undamped equilibrium state.

We performed a number of these simulations and selected a system from these with final (i.e. after turning off orbital damping) libration amplitude of $\sim 20^{\circ}$. Most simulations resulted in a Laplace argument with very small libration amplitude $\left(\sim 1^{\circ}\right)$ during the damping phase and rose to several tens of degrees after the damping was removed. This particular system had a libration amplitude significantly smaller than the best fit from Section 6, allowing us to test whether our fitting procedure artificially increases the libration amplitude. This synthetic system will be referred to as

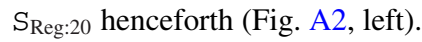

We generate an RV data set for both $S_{\text {Chaos:80 }}$ and $S_{\text {Reg:20 }}$ using the same RV time series with their associated offsets and jitters described in Section 3.2. We include the innermost, nonresonant planet with the following orbital properties and coplanar with the migrated planets: $P_{d}=1.937821 \mathrm{~d}, m_{d}=2.21 \times$ $10^{-5} \mathrm{M}_{\odot}, e_{d}=0.072, \omega_{d}=-137.09$, and $M_{d}=289.23$. For each synthetic measurement $\left(v_{\text {obs }}\right)$, we compute the RV value at that time $\left(v_{\text {mod }}\right)$, then add two noise terms: one drawn from a standard

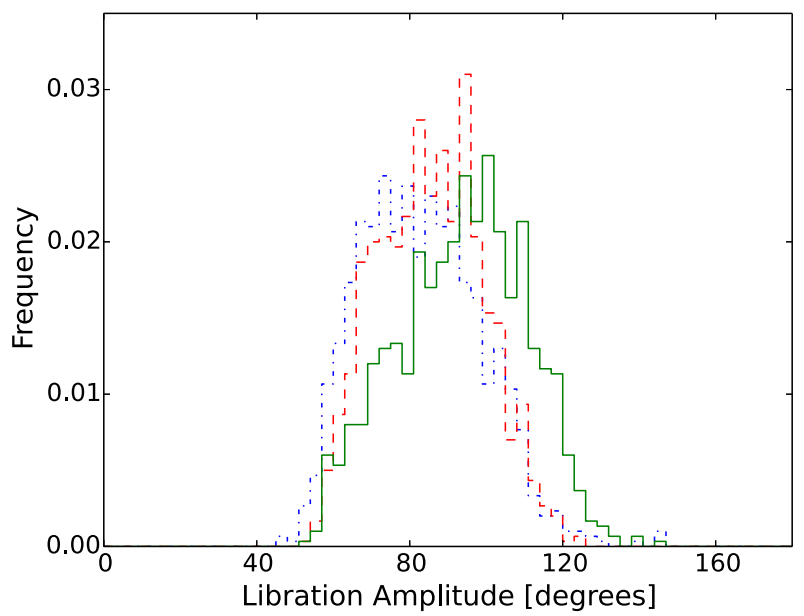

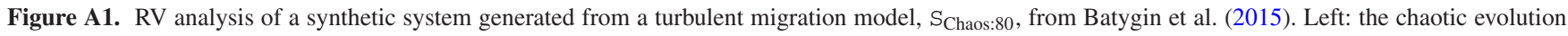
of $\phi_{\text {Laplace }}$ over the course of 100 years for model $\mathrm{S}_{\text {Chaos:80 }}$. Right: the distribution of the $\phi_{\text {Laplace }}$ libration amplitude based on three realizations (green solid, red dashed, blue dash-dotted) of the RV data generated from the synthetic system, $\mathrm{S}_{\text {Chaos:80. }}$. 

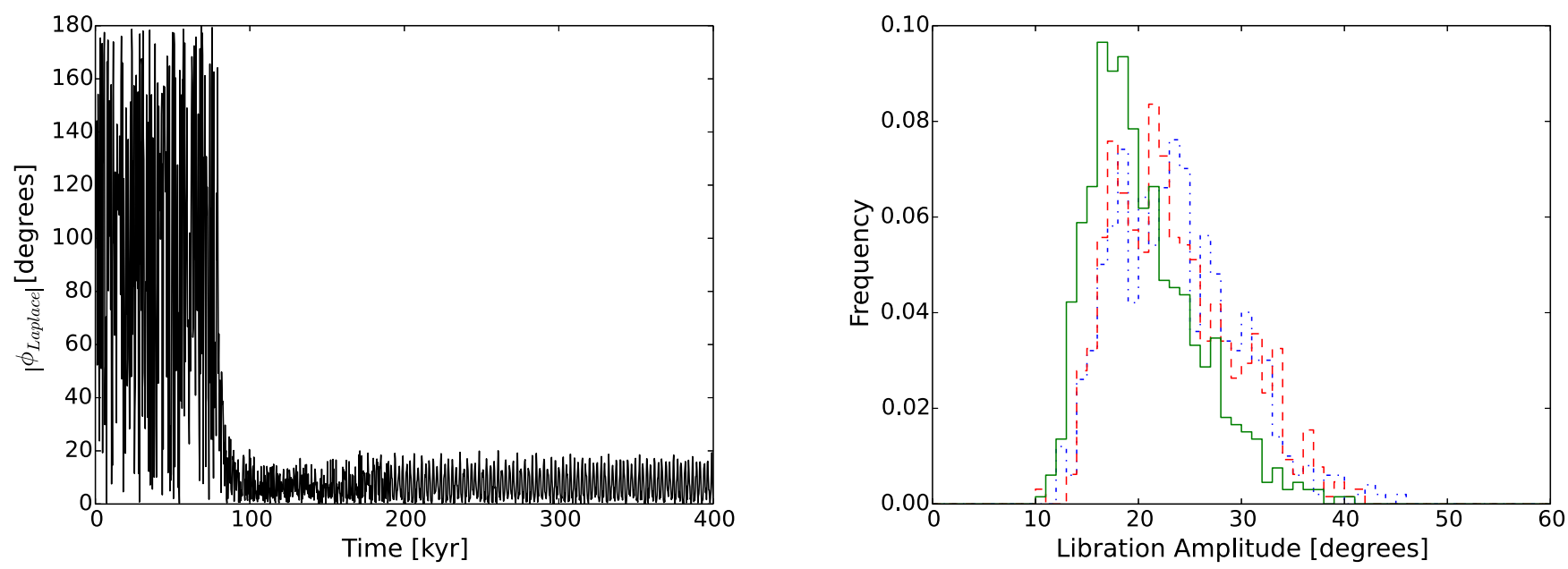

Figure A2. RV analysis of a synthetic system generated from a smooth migration model, $\mathrm{S}_{\text {Reg:20. }}$. Left: the evolution of the absolute value of the $\phi_{\text {Laplace }}$ over the course of $4 \times 10^{5}$ years for model $S_{\text {Reg:20 }}$. Right: the distribution of the $\phi_{\text {Laplace }}$ libration amplitude based on three realizations (green solid, red dashed, blue dash-dotted) of the RV data generated from the synthetic system, $\mathrm{S}_{\mathrm{Reg}: 20}$.

normal scaled by the measurement uncertainty $\sigma_{\mathrm{obs}}$ and a similar term but scaled by the jitter value corresponding to that observation, i.e. $v_{\text {obs }}=v_{\text {mod }}+\mathcal{N}(0,1) \times \sigma_{\text {obs }}+\mathcal{N}(0,1) \times \sigma_{\text {jit }}$.

These data sets were then used as simulated input observations for RUN DMC, and a full differential evolution MCMC was performed on each synthetic data set (in the same manner as was performed on the real data, and described in Sections 3.1 and 4). We performed three realizations of the RV time series for both $S_{\text {Chaos:80 }}$ and $S_{\text {Reg:20 }}$.

\section{A2 Results for synthetic planetary systems}

In right-hand panel of Fig. A1, we show the $\phi_{\text {Laplace }}$ libration amplitude distribution for the three realizations of the RV time series based on $S_{\text {Chaos:80 }}$. The chaotic nature of $S_{\text {Chaos:80 }}$ is shown in Fig. A1 (left) where the periodicity and peak-to-peak variation are not constant in time. There is a rough variation of $\sim 160^{\circ}$ corresponding to an amplitude of $\sim 80^{\circ}$. For each realization, we find libration amplitudes of $97 \pm_{19}^{14}$ (blue dash-dotted), $96 \pm_{21}^{16}$ (red dashed), and $91 \pm_{17}^{15}$ (green solid) degrees based on 1000 solutions each. Only a few solutions from each of these realizations were unstable over the short integration baseline (1 kyr). These estimates are qualitatively consistent with the input amplitude.

In right-hand panel of Fig. A2, we show the $\phi_{\text {Laplace }}$ libration amplitude distribution for the three realizations of the RV time series based on $\mathrm{S}_{\text {Chaos:80. A significant fraction of our posterior sample for }}$ each realization was not dynamically stable. Specifically, only 548 , 496, and 673 systems remained after the $1 \mathrm{kyr}$ integration. We find that the RV analysis of the synthetic data overestimated the outermost planet's mass by $\sim 20$ per cent, which could be reason for such a large fraction of unstable systems. We find libration amplitudes of $25.6 \pm_{5.3}^{4.7}$ (blue dash-dotted), $22.9 \pm_{5.5}^{6.8}$, (red dashed), and $19.5 \pm_{3.7}^{5.6}$ (green solid) degrees based on the remaining shortterm stable systems. Again, these values are qualitatively consistent with the input amplitude of $\sim 20^{\circ}$.

It is clear from these results that our RUN DMC algorithm does not significantly bias the recovered libration amplitudes for the $4: 2: 1$ resonance of the outer three planets in the GJ 876 system. Thus, we believe that our best-fitting libration amplitude for the real data (Section 6) is accurate and the GJ 876 system is in a chaotically librating state, with a high-amplitude libration.

\section{A3 Estimation of Lyapunov times for GJ 876 orbital solutions}

Batygin et al. (2015) suggest that the GJ 876 system is chaotic, with a characteristic time-scale (or Lyapunov time, defined below) for the chaos of roughly $14 \mathrm{yr}$. This theoretical study ignored the innermost planet and treated the system as coplanar. An estimate of the Lyapunov time for the four-planet, coplanar RV orbital solution of Rivera et al. (2010) was consistent with the analytic estimate. Barnes et al. (2015) suggest that mutually inclined systems in or near an MMR can also exhibit chaotic evolution.

Here we study the nature of three sets of solutions: first, the 1000 long-term stable, 3D orbital models described in Section 5; 1000 coplanar models described in Section 4; and 1000 coplanar models based on the $S_{\text {Reg:20 }}$. We determine whether or not the orbits are chaotic by evolving simultaneously the standard gravitational equations of motion and the variational equations of motion, which yields an estimate of the Lyapunov time of a trajectory (e.g. Lichtenberg \& Lieberman 1992). The variational equations govern the behaviour of small perturbations to an orbit and therefore can be used to study how perturbations evolve in time. For chaotic orbits, small perturbations of length $D$ grow exponentially as $D \sim \mathrm{e}^{t / \tau}$ with a characteristic time $\tau$, which in the limit as $t \rightarrow \infty$ is defined as the (minimum) Lyapunov time. Our finite time integrations are used to estimate this Lyapunov time as $t_{\text {final }} / \log \left[\left(D\left(t_{\text {final }}\right)\right]\right.$, where $t_{\text {final }}$ is the total integration time, $D(t=0)=1$, and $D\left(t_{\text {final }}\right)$ is the total length of the 'perturbation' at the end of the integration. Note that $D$ in principle can become very large, but since the variational equations are linear in the components of $D$, the absolute length of $D$ need not be small for the variational equations to apply. If an orbit is regular, the reported Lyapunov time will be comparable to the integration time, though integrations cannot prove that an orbit is regular. These integrations must therefore be carried out for long enough such that the chaotic and regular orbits have markedly different reported Lyapunov times.

We employed a Wisdom-Holman mapping in canonical astrocentric coordinates to integrate both the equations of motion and the variational equations (Wisdom \& Holman 1991). A third-order symplectic corrector was implemented to improve the accuracy of these integrations (Wisdom, Holman \& Touma 1996; Chambers 1999; Wisdom 2006). We used a simple prescription for the effects of general relativity which leads to precession of the orbits 
with the correct time-scale (Milani \& Nobili 1983). As we will show, the Lyapunov times of the orbits were significantly shorter than the time-scale of precession due to general relativity, and so this approximate version of general relativity is sufficient for a good estimate of the Lyapunov times for the orbits studied.

The Wisdom-Holman integrator can be used with a time step as large as a 10th or 20th of the shortest orbital time-scale in the system (Wisdom \& Holman 1992; Rauch \& Holman 1999). For GJ 876 , this corresponds to the time needed to resolve the pericentre passage of the innermost planet. We estimate this using the orbital period of the innermost planet as if its semimajor axis was equal to the pericentre distance, $P_{\text {eff }} \sim P_{\text {orb }}(1-e)^{3 / 2}=1.6 \mathrm{~d}$, where $e \approx 0.1$ and $P_{\text {orb }} \approx 2 \mathrm{~d}$. We used a time step of either 0.14 or $0.014 \mathrm{~d}$ when integrating the set of 3D solutions, and, as discussed below, these yield similar estimates of the Lyapunov times. For both coplanar set of solutions, we used a time step of $0.14 \mathrm{~d}$. The maximum fractional energy error $\Delta E / E$ was $\sim 10^{-5}$ for the $3 \mathrm{D}$ set of orbits with a time step of $0.14 \mathrm{~d}, \sim 10^{-10}$ for the same set with a time step of $0.014 \mathrm{~d}$, and $\sim 10^{-7}$ for the coplanar set of orbits. These integrations lasted $10^{4}$ years.

In Fig. A3, we show the distribution of Lyapunov times for the $3 \mathrm{D}$ set of solutions resulting from the integrations employing both time steps and the distribution of Lyapunov times for the coplanar set of solutions. These integrations all agree that the motion of these orbits is chaotic with a Lyapunov time of roughly $10 \mathrm{yr}$, consistent with the analytic estimate of Batygin et al. (2015). The small peak at $10^{3}$ years in the distribution of Lyapunov times for the coplanar orbital solutions to the data corresponds to orbits which might be regular. However, the vast majority of coplanar orbits studied were chaotic. The close agreement between Lyapunov times estimated for the 3D and coplanar sets is also consistent with the analysis of Batygin et al. (2015) and Martí et al. (2013) in that the chaotic motion is captured by a coplanar approximation of the true system.

During the integrations with a time step of $0.14 \mathrm{~d}, \sim 10$ orbits in each set (3D versus coplanar) were flagged as unstable. With the smaller time step of $0.014 \mathrm{~d}$, no orbit in the set of $3 \mathrm{D}$ orbits

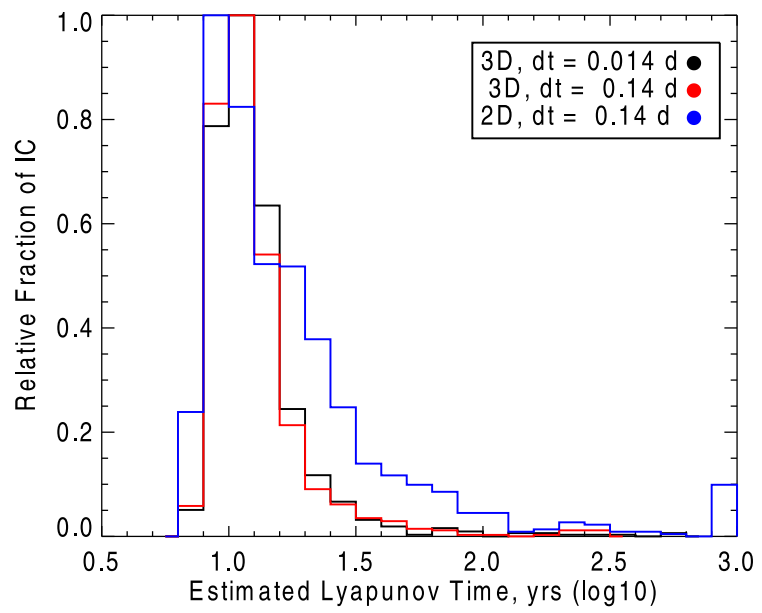

Figure A3. The distribution of estimated Lyapunov times for the GJ 876 system. These estimates are the result of integrations of the set of $10^{3} 3 \mathrm{D}$ orbital solutions fitted to the RV data, employing a time step of $0.14 \mathrm{~d}$ (red) and with a time step of $0.014 \mathrm{~d}$ (black), and for the 1000 solutions fitted to the data assuming coplanar orbits, which employed a time step of $0.14 \mathrm{~d}$ (blue).

was flagged as unstable. This suggests that the smaller time step might be necessary for studying the long-term stability of these orbits using a Wisdom-Holman integrator. However, since the two time steps agree on the distribution of Lyapunov times, we believe that these results are robust. Lastly, we do not show the results of the coplanar set of solutions fitted to synthetic data, since our integrations suggested that nearly all of these orbits were not only chaotic but also showed instability on short $\left(10^{4} \mathrm{yr}\right)$ time-scales. We defer any further analysis of the stability of these synthetic solutions to future work.

This paper has been typeset from a $\mathrm{T}_{\mathrm{E}} \mathrm{X} / \mathrm{L} \mathrm{T} \mathrm{E} \mathrm{X}$ file prepared by the author. 